\title{
SOME THIRD-ORDER IRREGULAR BOUNDARY VALUE PROBLEMS*
}

BY

\author{
LEWIS E. WARD
}

\section{INTRODUCTION}

We treat here the problem of expanding functions in infinite series whose terms are solutions of the differential equation $d^{3} u / d x^{3}+\rho^{3} u=0$ and various types of boundary conditions. Birkhoff $\dagger$ has treated a general problem of this nature for the case in which the boundary conditions are regular, and Hopkins $\ddagger$ has considered a very special case of irregularity. The first part of this paper is an extension of Hopkins' work in which the distinctive feature of the boundary conditions is that two bear at only one point and the third bears at two points. In the second part of the paper is considered a very special case in which one boundary condition bears at one point, the second at another point, which is complex, and the third at both points. And in the third part is considered a case in which the boundary conditions bear symmetrically at three points, one at each point.

\section{PART I}

The boundary conditions are written $W_{i}(u)=0, i=1,2,3$, where $W_{i}(u)$ is a linear homogeneous form in $u(a), u^{\prime}(a), u^{\prime \prime}(a), u(b), u^{\prime}(b), u^{\prime \prime}(b)$ with constant coefficients, which we shall suppose to be real. Here $a$ and $b$ are taken real and positive and $a<b$. By linear combination among themselves the conditions can be reduced to the equivalent set

$$
\begin{aligned}
& \alpha_{12} u^{\prime \prime}(a)+\alpha_{11} u^{\prime}(a)+\alpha_{10} u(a) \quad+\beta_{11} u^{\prime}(b)+\beta_{10} u(b)=0, \\
& \alpha_{22} u^{\prime \prime}(a)+\alpha_{21} u^{\prime}(a)+\alpha_{20} u(a)+\beta_{22} u^{\prime \prime}(b)+\beta_{21} u^{\prime}(b)+\beta_{20} u(b)=0 \text {, } \\
& \alpha_{31} u^{\prime}(a)+\alpha_{30} u(a)+\beta_{31} u^{\prime}(b)+\beta_{30} u(b)=0 \text {. }
\end{aligned}
$$

We shall represent by $W_{1}(u), W_{2}(u)$, and $W_{3}(u)$ the left hand sides of these three equations respectively, by $W_{i a}(u)$ that portion of $W_{i}(u)$ which bears at $a$, and by $W_{i b}(u)$ that portion which bears at $b$.

\footnotetext{
* Presented to the Society, April 16, 1927; received by the editors in October, 1926.

$\dagger$ G. D. Birkhoff, these Transactions, vol. 9 (1908), pp. 219-231 and pp. 373-395.

$\ddagger$ J. W. Hopkins, published by D. Jackson in these Transactions, vol. 20 (1919), p. 245, et seq.
} 
The characteristic equation is $\Delta(\rho)=0$, where

$$
\Delta(\rho) \equiv\left|\begin{array}{lll}
W_{1}\left(y_{1}\right) & W_{1}\left(y_{2}\right) & W_{1}\left(y_{3}\right) \\
W_{2}\left(y_{1}\right) & W_{2}\left(y_{2}\right) & W_{2}\left(y_{3}\right) \\
W_{3}\left(y_{1}\right) & W_{3}\left(y_{2}\right) & W_{3}\left(y_{3}\right)
\end{array}\right| .
$$

Here $y_{1}, y_{2}$, and $y_{3}$ are any three linearly independent solutions of the differential equation. We shall take

$$
y_{i}=e^{\omega_{i} \rho(x-a)} \text {, where } \omega_{1}=-1, \omega_{2}=e^{\pi i / 3}, \omega_{3}=e^{-\pi i / 3} .
$$

Inserting these functions and expanding the determinant, we find

$$
\begin{aligned}
\Delta(\rho)= & \Delta_{a a a}+\Delta_{a a b} e^{\omega_{2} \rho(b-a)}+\Delta_{a b a} e^{\omega_{2} \rho(b-a)}+\Delta_{b a a} e^{\omega_{1} \rho(b-a)} \\
& +\Delta_{b b b}+\Delta_{b b a} e^{\omega_{\rho} \rho(a-b)}+\Delta_{b a b} e^{\omega_{2} \rho(a-b)}+\Delta_{a b b} e^{\omega_{1} \rho(a-b)},
\end{aligned}
$$

where

$$
\begin{aligned}
& \Delta_{a a a}=3\left(\omega_{2}-\omega_{3}\right) \rho^{3} D_{a}, \\
& \Delta_{a a b}=\left(\omega_{1}-\omega_{2}\right) \rho\left[-\omega_{3} D_{a 4} \rho^{4}-\omega_{1} D_{a 3} \rho^{3}-\omega_{2} D_{a 2 \rho^{2}}-\omega_{3} D_{a 1} \rho-\omega_{1} D_{a 0}\right], \\
& \Delta_{a b a}=\left(\omega_{3}-\omega_{1}\right) \rho\left[-\omega_{2} D_{a 4} \rho^{4}-\omega_{1} D_{a 3} \rho^{3}-\omega_{3} D_{a 2 \rho^{2}}-\omega_{2} D_{a 1} \rho-\omega_{1} D_{a 0}\right], \\
& \Delta_{b a a}=\left(\omega_{2}-\omega_{3}\right) \rho\left[D_{a 4} \rho^{4}+D_{a 3 \rho^{3}}+D_{a 2 \rho^{2}}+D_{a 1 \rho}+D_{a 0}\right], \\
& \Delta_{a b b}=\left(\omega_{2}-\omega_{3}\right) \rho\left[D_{b 4 \rho^{4}}+D_{b 3} \rho^{3}+D_{b 2 \rho^{2}}+D_{b 1} \rho+D_{b 0}\right] \\
& \Delta_{b a b}=\left(\omega_{3}-\omega_{1}\right) \rho\left[-\omega_{2} D_{b 4 \rho^{4}}-\omega_{1} D_{b 3 \rho^{3}}-\omega_{3} D_{b 2 \rho} \rho^{2}-\omega_{2} D_{b 1 \rho}-\omega_{1} D_{b 0}\right], \\
& \Delta_{b b a}=\left(\omega_{1}-\omega_{2}\right) \rho\left[-\omega_{3} D_{b 4} \rho^{4}-\omega_{1} D_{b 3 \rho^{3}}-\omega_{2} D_{b 2 \rho^{2}}-\omega_{3} D_{b 1 \rho}-\omega_{1} D_{b 0}\right], \\
& \Delta_{b b b}=3\left(\omega_{2}-\omega_{3}\right) \rho^{3} D_{b} .
\end{aligned}
$$

In these expressions the $D$ 's are certain real combinations of determinants formed from the array of coefficients in the boundary conditions.

The regularity condition, which bears on the differential system, plays an important rôle in boundary value and expansion problems. In the present case the system is regular if each of the six exponentials in $\Delta(\rho)$ is present and multiplied by a polynomial of degree five in $\rho$. As mentioned in the introduction the regular case has been treated by Birkhoff. The cases in which the multiplying polynomials are of degrees less than five are susceptible of a treatment similar to that employed by Birkhoff, and the results are similar to those of the regular case. They will not be considered here.

We propose to treat some of the cases in which every $D_{a}$ equals zero or every $D_{b}$ equals zero. These we call the highly irregular cases. One of them is Hopkins' case. For the sake of definiteness we shall take $D_{b i}=0$, $i=0,1,2,3,4$. 
The characteristic equation. Divide the $\rho$ plane into six equal sectors defined by $l \pi / 3<\arg \rho<(l+1) \pi / 3, l=0,1,2,3,4,5$. It is only near the rays $\arg \rho=0,2 \pi / 3,4 \pi / 3$ that we can hope to find characteristic numbers, for if $|\rho|$ is large and $\rho$ is not near one of these rays in an angular sense, some one of the exponential terms in $\Delta(\rho)$ will far outbalance in numerical value all the others. But since $\rho$ appears in the differential equation only as $\rho^{3}$, we may restrict $\rho$ to the sector $-\pi / 3 \leqq \arg \rho \leqq \pi / 3$.

Consequently we need look for characteristic numbers only near the ray $\arg \rho=0$.

We may write

where

$$
\Delta(\rho)=e^{\omega_{s} \rho(b-a)}[\phi(\rho)+\epsilon(\rho)],
$$

$$
\phi(\rho)=\Delta_{a a b}+\Delta_{a b a} e^{\left(\omega_{2}-\omega_{3}\right) \rho(b-a)}
$$

and

$$
\epsilon(\rho)=\left(\Delta_{a a a}+\Delta_{b b b}\right) e^{\omega_{3} \rho(a-b)}+\Delta_{b a a} e^{\left(\omega_{1}-\omega_{z}\right) \rho(b-a)} .
$$

If we lay down the further restriction $-\pi / 6+\delta \leqq \arg \rho \leqq \pi / 3, \delta$ being a small positive constant, $|\epsilon(\rho)|$ will be small for $|\rho|$ large. If $D_{a j}$ is that $D_{a}$ having the largest second subscript and not being zero, we have

$$
\begin{aligned}
\phi(\rho) & =\left(\omega_{1}-\omega_{2}\right) \sum_{k=0}^{j} \omega_{k+1}^{2} D_{a k} \rho^{k+1}+\left(\omega_{2}-\omega_{1}\right) e^{\left(\omega_{2}-\omega_{3}\right) \rho(b-a)} \sum_{k=0}^{j} \omega_{k} D_{a k} \rho^{k+1} \\
& =\left(\omega_{1}-\omega_{2}\right) \rho^{j+1}\left[\omega_{j} D_{a j}+\left(\frac{1}{\rho}\right)\right]\left[\psi(\rho)+\epsilon^{\prime}(\rho)\right],
\end{aligned}
$$

where $(1 / \rho)$ means a quantity of the size of $1 / \rho$ for $|\rho|$ large, $\left|\epsilon^{\prime}(\rho)\right|$ is small for $|\rho|$ large, and

$$
\psi(\rho)=\frac{\omega_{j+1}^{2}}{\omega_{j}}-e^{\left(\omega_{3}-\omega_{3}\right) \rho(b-a)} .
$$

Here $\omega_{0}=\omega_{3}, \omega_{4}=\omega_{1}$, and $\omega_{5}=\omega_{2}$.

We have

$$
\psi(\rho)=\frac{\omega_{j+1}^{2}}{\omega_{j}}-e^{i \rho 3^{1 / 2}(b-a)},
$$

whose zeros are given by

$$
\rho=\frac{1}{i \cdot 3^{1 / 2}(b-a)} \log \frac{\omega_{j}^{2}+1}{\omega_{j}} .
$$

This logarithm has one of the values $\pi i / 3,-\pi i / 3$, or $\pi i, j$ being $0,1,2,3$, or 4. Hence the zeros of $\psi(\rho)$ are real and are evenly spaced along the ray $\arg \rho=0$. 
We can now discuss the zeros of $\Delta(\rho)$. For we have

$$
\Delta(\rho)=\left(\omega_{1}-\omega_{2}\right) \rho^{i+1} e^{\omega_{p} \rho(b-a)}\left[\omega_{j} D_{a j}+\left(\frac{1}{\rho}\right)\right]\left[\psi(\rho)+\epsilon^{\prime \prime}(\rho)\right],
$$

where $\left|\epsilon^{\prime \prime}(\rho)\right|$ is small for $|\rho|$ large. Draw small circles all of the same radius centered at the zeros of $\psi(\rho)$, and call $S$ the portion of the sector $(-\pi / 6$ $+\delta, \pi / 3)$ which is exterior to these circles. As the imaginary part of $\rho$ becomes positively infinite, $\psi(\rho) \rightarrow \omega_{i+1}^{2} / \omega_{j}$; as the imaginary part of $\rho$ becomes negatively infinite, $\psi(\rho) \rightarrow \infty$; and $\psi(\rho)$ has a real period. Hence $|\psi(\rho)|$ has a positive minimum $\mu$ in $S$. Hence from (1)

$$
|\Delta(\rho)|>\left|\left(\omega_{1}-\omega_{2}\right) \rho^{j+1} e^{\omega_{\rho} \rho(b-a)}\left[\omega_{j} D_{a j}+\left(\frac{1}{\rho}\right)\right]\right| \cdot|\mu-| \epsilon^{\prime \prime}(\rho)|| .
$$

Now let $|\rho|$ be so large that $\left|\epsilon^{\prime \prime}(\rho)\right|<\mu / 2$. Then we have

$$
|\Delta(\rho)|>h\left|\rho^{i+1} e^{\operatorname{mas}(b-a)}\right| \text {, }
$$

and this inequality is valid for $\rho$ in $S$ and $|\rho|$ sufficiently large, $h$ being independent of $\rho$. This inequality shows that $\Delta(\rho)$ has no zeros in $S$ for $|\rho|$ large.

That $\Delta(\rho)$ for large $|\rho|$ does have a zero in each small circle and only one is seen from (1). As $\rho$ travels just once around one of the circles, $\arg \psi(\rho)$ increases by just $2 \pi$, i.e., the point $\psi(\rho)$ travels just once around the origin. Hence the point $\Delta(\rho)$ will travel just once around the origin provided $\left|\epsilon^{\prime \prime}(\rho)\right|$ is small enough, and hence $\Delta(\rho)$ has just one zero in the circle. Moreover, if $|\rho|$ is large enough, the zeros of $\Delta(\rho)$ will be real. We omit the actual proof, which consists in showing by purely formal manipulations that $\overline{\Delta(\rho)}=-\Delta(\bar{\rho})$, dashes denoting conjugates. We denote the zeros of $\Delta(\rho)$ by $\rho_{k}, k=1,2, \cdots$.

The characteristic functions. A form of solution of the differential equation and the first and third boundary conditions is

$$
u(x)=\frac{1}{\left(\omega_{2}-\omega_{3}\right) \rho}\left|\begin{array}{ccc}
y_{1}(x) & y_{2}(x) & y_{3}(x) \\
W_{1}\left(y_{1}\right) & W_{1}\left(y_{2}\right) & W_{1}\left(y_{3}\right) \\
W_{3}\left(y_{1}\right) & W_{3}\left(y_{2}\right) & W_{3}\left(y_{3}\right)
\end{array}\right| .
$$

Substitution of $\rho_{k}$ for $\rho$ in $u(x)$ yields the $k$ th characteristic function $u_{k}(x)$. We consider the characteristic functions for the case in which

(3) $W_{2}(u)=\alpha_{22} u^{\prime \prime}(a)+\alpha_{21} u^{\prime}(a)+\alpha_{20} u(a)+\beta_{22} u^{\prime \prime}(b)+\beta_{21} u^{\prime}(b)+\beta_{20} u(b)$,

$$
W_{3}(u)=\quad \alpha_{31} u^{\prime}(a)+\alpha_{30} u(a),
$$


the distinctive feature of which is that two of the $W$ 's bear only at one end of the interval. This is a case in which every $D_{b}$ equals zero. Highly irregular cases in which at least two boundary conditions bear at both ends of the interval will be treated in a later paper.

Proceeding to develop the form of the characteristic functions, we have $u(x)=A_{1}(\rho) y_{1}(x)+A_{2}(\rho) y_{2}(x)+A_{3}(\rho) y_{3}(x)$, where the $A$ 's are certain quadratic polynomials in $\rho$. We may write

$$
\begin{gathered}
u(x)=\alpha_{12} \alpha_{31} \rho^{2} \delta_{1}[\rho(x-a)]+\alpha_{12} \alpha_{30} \rho \delta_{2}[\rho(x-a)] \\
+\left(\alpha_{11} \alpha_{30}-\alpha_{10} \alpha_{31}\right) \delta_{3}[\rho(x-a)]
\end{gathered}
$$

where

$$
\begin{aligned}
& \delta_{1}(\rho t)=e^{\omega_{1} \rho t}+e^{\omega_{2} \rho t}+e^{\omega_{2} \rho t}=e^{-\rho t}+2 e^{\rho t / 2} \cos \left(\frac{3^{1 / 2}}{2} \rho t\right), \\
& \delta_{2}(\rho t)=e^{\omega_{1} \rho t}-\omega_{3} e^{\omega_{2} \rho t}-\omega_{2} e^{\omega_{2} \rho t}=e^{-\rho t}-2 e^{\rho t / 2} \cos \left(-\frac{\pi}{3}+\frac{3^{1 / 2}}{2} \rho t\right), \\
& \delta_{3}(\rho t)=e^{\omega_{1} \rho t}-\omega_{2} e^{\omega_{2} \rho t}-\omega_{3} e^{\omega_{2} \rho t}=e^{-\rho t}-2 e^{\rho t / 2} \cos \left(\frac{\pi}{3}+\frac{3^{1 / 2}}{2} \rho t\right) .
\end{aligned}
$$

These $\delta$ functions are seen to be real if $\rho$ and $t$ are real, and they satisfy the relations

$$
\begin{gathered}
\frac{d}{d t} \delta_{1}(\rho t)=-\rho \delta_{3}(\rho t), \quad \frac{d}{d t} \delta_{2}(\rho t)=-\rho \delta_{1}(\rho t), \quad \frac{d}{d t} \delta_{3}(\rho t)=-\rho \delta_{2}(\rho t) ; \\
\delta_{1}(0)=3, \quad \delta_{2}(0)=0, \quad \delta_{3}(0)=0, \text { etc. }
\end{gathered}
$$

Hence $u(x)$ is real if both $\rho$ and $x$ are real. We may write

$$
\begin{aligned}
u(x)= & e^{-\rho(x-a)}\left[\alpha_{12} \alpha_{31} \rho^{2}+\alpha_{12} \alpha_{30 \rho}+\left(\alpha_{11} \alpha_{30}-\alpha_{10} \alpha_{31}\right)\right] \\
+ & 2 e^{\rho(x-a) / 2}\left[\alpha_{12} \alpha_{31} \rho^{2} \cos \left\{\frac{3^{1 / 2}}{2} \rho(x-a)\right\}-\alpha_{12} \alpha_{30 \rho} \cos \left\{-\frac{\pi}{3}\right.\right. \\
& \left.\left.+\frac{3^{1 / 2}}{2} \rho(x-a)\right\}-\left(\alpha_{11} \alpha_{30}-\alpha_{10} \alpha_{31}\right) \cos \left\{\frac{\pi}{3}+\frac{3^{1 / 2}}{2} \rho(x-a)\right\}\right] .
\end{aligned}
$$

Necessary conditions for convergence of formal series. We state the following

LEMMA. If the series

$$
\sum_{k=1}^{\infty} a_{k} u_{k}(x)
$$


converges uniformly in some interval $\alpha \leqq x \leqq \beta$, where $0<\alpha<\beta<\pi$, then $\left|a_{k}\right|<\mu e^{-\rho_{k}\left(x_{0}-a\right) / 2}$, where $x_{0}$ is any constant less than $\beta$, and $\mu$ is independent of $k$.

Noting that at least one of the cosine terms in (5) must be present, for otherwise we should not have more than two boundary conditions (3), the proof of this lemma is the same as that of the corresponding one in Hopkins' paper.

In the series (6) we now allow $x$ to be complex. Writing $x-a=t$, we have

$$
\left|\delta_{i}\left[\rho_{k}(x-a)\right]\right|<\sum_{j=1}^{3}\left|e^{\omega j \rho_{k} t}\right| ;
$$

and, calling $t=\xi+\eta i, \xi$ and $\eta$ both being real,

$$
\left|\delta_{i}\left[\rho_{k}(x-a)\right]\right|<e^{-\rho_{k} \xi}+e^{\rho_{k}\left(\xi-\eta \cdot 3^{1 / 2}\right) / 2}+e^{\rho_{k}\left(\xi+\eta \cdot 3^{1 / 2}\right) / 2} .
$$

Here $k$ is supposed to be so large that $\rho_{k}$ is known to be real. Let $t_{0}=x_{0}-a$, and choose a number $x_{1}$ less than $x_{0}$ but greater than $a$. Let $t_{1}=x_{1}-a$. Then $t_{1}-t_{0}=x_{1}-x_{0}<0$. We shall have

$$
\left.\begin{array}{rl}
e^{-\rho k \xi} & <e^{\rho k t_{1} / 2} \\
e^{\rho k\left(\xi-\eta \cdot 3^{1 / 2}\right) / 2} & <e^{\rho t_{1} / 2} \\
e^{\rho_{k}\left(\xi+\eta \cdot 3^{1 / 2}\right) / 2} & <e^{\rho k t_{1} / 2}
\end{array}\right\} \text { provided }\left\{\begin{array}{l}
\xi>-\frac{1}{2} t_{1} \\
\xi-\eta \cdot 3^{1 / 2}<t_{1} \\
\xi+\eta \cdot 3^{1 / 2}<t_{1} .
\end{array}\right.
$$

All of these inequalities are satisfied if $t$ is within the triangle whose sides are $\xi=-t_{1} / 2, \xi-\eta \cdot 3^{1 / 2}=t_{1}, \xi+\eta \cdot 3^{1 / 2}=t_{1}$, i.e., if $x$ is within the equilateral triangle centered at $x=a$ and having one vertex at $x=x_{1}$. Hence we have within this triangle

$$
\left|u_{k}(x)\right|<3 e^{\rho k\left(x_{1}-a\right) / 2} \text { and }\left|a_{k} u_{k}(x)\right|<\frac{3 g}{h} e^{\rho k\left(x_{1}-x_{0}\right) / 2} .
$$

But the expression on the right is the general term of a convergent series of positive constants. Thus we have proved

TheOREM 1. Under the hypothesis of the above lemma series (6) converges uniformly in the interior of the equilateral triangle centered at $x=a$ and having one vertex at $x=x_{0}$, and represents therein an analytic function of the complex variable $x$.

This theorem presents a restriction on the type of function representable by series (6). But there are further restrictions. We may write $f(x)=\sum_{k=0}^{\infty} c_{k}(x-a)^{k}=\sum_{k=1}^{\infty} a_{k} u_{k}(x)$, where $c_{k}=(1 / k !) f^{(k)}(a)$. By the first of 
(3) we have $\alpha_{12} u_{k}^{\prime \prime}(a)+\alpha_{11} u_{k}^{\prime}(a)+\alpha_{10} u_{k}(a)=0$. Use of the differential equation satisfied by $u_{k}(x)$ gives

$$
\alpha_{12} u_{k}^{(2+8 n)}(a)+\alpha_{11} u_{k}^{(1+3 n)}(a)+\alpha_{10} u_{k}^{(3 n)}(a)=0(n=0,1,2, \cdots) .
$$

Hence

$$
\alpha_{12} f^{(2+3 n)}(a)+\alpha_{11} f^{(1+3 n)}(a)+\alpha_{10} f^{(3 n)}(a)=0 \quad(n=0,1,2, \cdots) .
$$

Hence

$$
\begin{aligned}
& \alpha_{12}(2+3 n)(1+3 n) c_{2+3 n}+\alpha_{11}(1+3 n) c_{1+3 n}+\alpha_{10} c_{8 n}=0 \\
&(n=0,1,2, \cdots) .
\end{aligned}
$$

Now write $f(x)=\phi_{1}(x)+\phi_{2}(x)+\phi_{3}(x)$, where

$$
\begin{gathered}
\phi_{1}(x)=\sum_{n=0}^{\infty} c_{3 n}(x-a)^{3 n}, \quad \phi_{2}(x)=\sum_{n=0}^{\infty} c_{1+8 n}(x-a)^{1+3 n}, \\
\phi_{3}(x)=\sum_{n=0}^{\infty} c_{2+3 n}(x-a)^{2+3 n},
\end{gathered}
$$

and form the expression

$$
\begin{aligned}
& \alpha_{12} \phi_{8}{ }^{\prime \prime}(x)+\alpha_{11} \phi_{2}^{\prime}(x)+\alpha_{10} \phi_{1}(x)=\alpha_{12} \sum_{n=0}^{\infty} c_{2+3 n}(2+3 n)(1+3 n)(x-a)^{3 n} \\
& +\alpha_{11} \sum_{n=0}^{\infty} c_{1+3 n}(1+3 n)(x-a)^{3 n} \\
& +\alpha_{10} \sum_{n=0}^{\infty} c_{3 n}(x-a)^{3 n} \\
& =\sum_{n=0}^{\infty}\left[\alpha_{12}(2+3 n)(1+3 n) c_{2+3 n}+\alpha_{11}(1+3 n) c_{1+3 n}+\alpha_{10} c_{3 n}\right](x-a)^{3 n} .
\end{aligned}
$$

By (7) the coefficient of every power of $(x-a)$ in this series is seen to equal zero. Hence

$$
\alpha_{12} \phi_{8}^{\prime \prime}(x)+\alpha_{11} \phi_{2}^{\prime}(x)+\alpha_{10} \phi_{1}(x) \equiv 0 .
$$

Similarly

$$
\alpha_{31} \phi_{2}^{\prime}(x)+\alpha_{30} \phi_{1}(x) \equiv 0 .
$$

Convergence proof. The conditions of analyticity at $x=a$ and identities (8) together with certain auxiliary conditions imposed on $f(x)$ for the purpose of accomplishing readily the convergence proof constitute sufficient conditions that $f(x)$ be expansible in a series of type (6). The convergence proof will now be given, and the theorem stated after the proof is finished. 
We introduce a Green's function and a contour integral equal to the sum $I_{n}(x)$ of the first $n$ terms of the formal series arising from $f(x) .{ }^{*}$ Let

$$
\begin{aligned}
g(x, s, \rho) & = \pm \frac{1}{2}\left|\begin{array}{lll}
y_{1}(x) & y_{2}(x) & y_{3}(x) \\
y_{1}^{\prime}(s) & y_{2}^{\prime \prime}(s) & y_{3}^{\prime}(s) \\
y_{1}(s) & y_{2}(s) & y_{3}(s)
\end{array}\right| \div\left|\begin{array}{lll}
y_{1}^{\prime \prime}(s) & y_{2}^{\prime \prime}(s) & y_{3}^{\prime \prime}(s) \\
y_{1}^{\prime}(s) & y_{2}^{\prime}(s) & y_{3}^{\prime}(s) \\
y_{1}(s) & y_{2}(s) & y_{3}(s)
\end{array}\right| \\
& = \pm \frac{1}{2} \sum_{1}^{s} y_{i}(x) z_{i}(s)= \pm \frac{1}{6 \rho^{2}} \delta_{3}[\rho(x-s)], \quad\left\{\begin{array}{l}
+ \text { if } x>s \\
- \text { if } x<s .
\end{array}\right.
\end{aligned}
$$

Here the definitions of the functions $z_{1}(s)$ are obvious, and the reduction to the $\delta$ function is straightforward. It turns out that

Let

$$
z_{i}(s)=-\frac{\omega_{i}}{3 \rho^{2}} e^{\omega_{i} \rho(a-s)} .
$$

Then

$$
G(x, s, \rho)=-\frac{1}{\Delta(\rho)}\left|\begin{array}{llll}
y_{1}(x) & y_{2}(x) & y_{3}(x) & g(x, s, \rho) \\
W_{1}\left(y_{1}\right) & W_{1}\left(y_{2}\right) & W_{1}\left(y_{3}\right) & W_{1}(g) \\
W_{2}\left(y_{1}\right) & W_{2}\left(y_{2}\right) & W_{2}\left(y_{3}\right) & W_{2}(g) \\
W_{3}\left(y_{1}\right) & W_{3}\left(y_{2}\right) & W_{3}\left(y_{3}\right) & W_{3}(g)
\end{array}\right|
$$

$$
I_{n}(x)=\frac{1}{2 \pi i} \int_{\gamma_{n}} \int_{a}^{b} 3 \rho^{2} G(x, s, \rho) f(s) d s d \rho,
$$

where $\gamma_{n}$ is an arc of a circle centered at $\rho=0$ and extending from $\arg \rho=-\pi / 3$ to $\arg \rho=\pi / 3$. It is supposed that the radius of $\gamma_{n}$ is greater than $\left|\rho_{n}\right|$ but less than $\left|\rho_{n+1}\right|$.

Before considering the integration we alter the form of $G$. Multiplying the first, second, and third columns of the determinant in $G$ by $z_{1}(s)$, $\frac{1}{2} z_{2}(s)$, and $\frac{1}{2} z_{3}(s)$ respectively, and adding to the fourth column, we get

$$
G=-\frac{1}{\Delta(\rho)}\left|\begin{array}{lllc}
y_{1}(x) & y_{2}(x) & y_{3}(x) & g(x, s, \rho)+\frac{1}{2} \sum_{i=1}^{3} y_{i}(x) z_{i}(s) \\
W_{1}\left(y_{1}\right) & W_{1}\left(y_{2}\right) & W_{1}\left(y_{3}\right) & 0 \\
W_{2}\left(y_{1}\right) & W_{2}\left(y_{2}\right) & W_{2}\left(y_{3}\right) & 2 W_{2 b}(g) \\
W_{3}\left(y_{1}\right) & W_{3}\left(y_{2}\right) & W_{3}\left(y_{3}\right) & 0
\end{array}\right|,
$$

which on being expanded gives

$$
G=g+\frac{1}{2} \sum_{1}^{3} y_{i}(x) z_{i}(s)+\frac{2\left(\omega_{2}-\omega_{3}\right) \rho W_{2 b}(g)}{\Delta(\rho)} u(x) .
$$

- See Birkhoff, loc. cit., p. 379 and p. 390. 
Further, $\Delta(\rho)=\left(\omega_{2}-\omega_{3}\right) \rho\left[3 \rho^{2} D_{a}-W_{2 b}(u)\right]$, so that

$$
\begin{array}{ll}
G=\frac{1}{3 \rho^{2}} \delta_{3}[\rho(x-s)]+\frac{2 W_{2 b}(g) u(x)}{3 \rho^{2} D_{a}-W_{2 b}(u)} & \text { if } x>s, \\
G=\frac{2 W_{2 b}(g) u(x)}{3 \rho^{2} D_{a}-W_{2 b}(u)} & \text { if } x<s .
\end{array}
$$

Thus we obtain the form of contour integral with which it is convenient to work, namely,

$$
\begin{aligned}
I_{n}(x)= & \frac{1}{2 \pi i} \int_{\gamma_{n}} \int_{a}^{x} \delta_{3}[\rho(x-s)] f(s) d s d \rho \\
& +\frac{1}{2 \pi i} \int_{\gamma_{n}} \int_{a}^{b} 3 \rho^{2} \frac{2 W_{2 b}(g) u(x)}{3 \rho^{2} D_{a}-W_{2 b}(u)} f(s) d s d \rho .
\end{aligned}
$$

The following six functions are introduced :

$$
\begin{array}{r}
P_{1}(x)=\rho^{2} \phi_{1}(a) \delta_{2}[\rho(x-a)]-\rho \phi_{2}^{\prime}(a) \delta_{3}[\rho(x-a)]+\phi_{3}{ }^{\prime \prime}(a) \delta_{1}[\rho(x-a)], \\
P_{2}(x)=\rho^{2} \phi_{1}(a) \delta_{3}[\rho(x-a)]-\rho \phi_{2}^{\prime}(a) \delta_{1}[\rho(x-a)]+\phi_{3}{ }^{\prime \prime}(a) \delta_{2}[\rho(x-a)], \\
P_{3}(x)=\rho^{2} \phi_{1}(a) \delta_{1}[\rho(x-a)]-\rho \phi_{2}^{\prime}(a) \delta_{2}[\rho(x-a)]+\phi_{3}{ }^{\prime \prime}(a) \delta_{3}[\rho(x-a)], \\
R_{1}(x)=-\rho \int_{a}^{x} \delta_{3}[\rho(x-s)] \phi_{1}{ }^{\prime \prime}(s) d s+\int_{a}^{x} \delta_{1}[\rho(x-s)] \phi_{2}{ }^{\prime \prime \prime}(s) d s \\
-\frac{1}{\rho} \int_{a}^{x} \delta_{2}[\rho(x-s)] \phi_{\phi_{3}}{ }^{\mathrm{iv}}(s) d s \\
R_{2}(x)=-\rho \int_{a}^{x} \delta_{1}[\rho(x-s)] \phi_{1}{ }^{\prime \prime}(s) d s+\int_{a}^{x} \delta_{2}[\rho(x-s)] \phi_{2}{ }^{\prime \prime \prime}(s) d s \\
-\frac{1}{\rho} \int_{a}^{x} \delta_{3}[\rho(x-s)] \phi_{3}{ }^{\mathrm{iv}}(s) d s \\
R_{3}(x)=-\rho \int_{a}^{x} \delta_{2}[\rho(x-s)] \phi_{1}{ }^{\prime \prime}(s) d s+\int_{a}^{x} \delta_{3}[\rho(x-s)] \phi_{2}{ }^{\prime \prime \prime}(s) d s \\
-\frac{1}{\rho} \int_{a}^{x} \delta_{1}[\rho(x-s)] \phi_{3}{ }^{\mathrm{iv}}(s) d s .
\end{array}
$$

Using $f(x)=\phi_{1}(x)+\phi_{2}(x)+\phi_{3}(x)$, and integrating by parts the term containing $\phi_{1}(s)$ twice, that containing $\phi_{2}(s)$ three times, and that containing $\phi_{\mathbf{3}}(s)$ four times, we find that

$$
\int_{a}^{x} \delta_{3}[\rho(x-s)] f(s) d s=\frac{3}{\rho} f(x)-\frac{3}{\rho^{4}} \phi_{3}^{\prime \prime}(x)-\frac{1}{\rho^{3}}\left\{P_{3}(x)+R_{3}(x)\right\},
$$


and

$$
\begin{aligned}
3 \rho^{2} \int_{a}^{b} 2 W_{2 b}(g) f(s) d s= & \beta_{22}\left[\frac{3}{\rho}\left\{\phi_{2}{ }^{\prime \prime}(b)+\phi_{3}{ }^{\prime \prime}(b)\right\}-\frac{1}{\rho}\left\{P_{1}(b)+R_{1}(b)\right\}\right] \\
& +\beta_{21}\left[\frac{3}{\rho} f^{\prime}(b)+\frac{1}{\rho^{2}}\left\{P_{2}(b)+R_{2}(b)\right\}\right] \\
+\beta_{20} & {\left[\frac{3}{\rho}\left\{f(b)-\frac{\phi_{3}{ }^{\prime \prime \prime}(b)}{\rho^{3}}\right\}-\frac{1}{\rho^{3}}\left\{P_{3}(b)+R_{3}(b)\right\}\right] . }
\end{aligned}
$$

These results are to be substituted in (9), and the terms separated into three groups, one containing $f(x)$, the second containing $\phi_{3}^{\prime \prime \prime}(x)$ and terms involving the values of the $\phi$ 's and their derivatives at $x=b$, and the third containing terms involving the $P$ 's and $R$ 's.

The $\rho$-integration is taken in two steps, one being an integration over $\gamma_{n}^{\prime}$, which is that part of $\gamma_{n}$ on which $\arg \rho>0$, and the other an integration over $\gamma_{n}^{\prime \prime}$, on which $\arg \rho<0$. We consider first the integration over $\gamma_{n}^{\prime}$.

Since the inequality $|u(x)|<l\left|\rho^{2} e^{\omega_{3} \rho(x-a)}\right|$, where $l$ is independent of $\rho$, and inequality (2) are both valid on $\gamma_{n}^{\prime}$, we have

$$
\left|\frac{u(x)}{3 \rho^{2} D_{a}-W_{2 b}(u)}\right|<l \cdot 3^{1 / 2}\left|\frac{\rho^{3} e^{\omega_{3} \rho(x-a)}}{\Delta(\rho)}-\right|<\frac{l \cdot 3^{1 / 2}}{h}-\left|\rho^{2-j} e^{\omega_{3} \rho(x-b)}\right| .
$$

The exponential in the last expression has an exponent whose real part is negative over the entire $\operatorname{arc} \boldsymbol{\gamma}_{n}^{\prime}$. For this reason the second group of terms arising from (9) yields in the $\rho$-integration over $\gamma_{n}{ }^{\prime}$ only terms which tend uniformly to zero as $n \rightarrow \infty, x$ being restricted to a closed interval interior to $(a, b)$ not containing either $a$ or $b$. We shall refer to terms arising from (9) which tend to zero in this manner as terms which can be neglected.

The terms of group three are all put over the denominator $3 \rho^{2} D_{a}-W_{2 b}(u)$, and it is then seen that that part of the numerator which contains $D_{a}$ leads to terms which can be neglected. The terms of the numerator containing $P$ 's are

$$
\begin{aligned}
\frac{1}{\rho^{3}}\left[\beta_{22}\left\{P_{3}(x) u^{\prime \prime}(b)-\rho^{2} P_{1}(b) u(x)\right\}+\right. & \beta_{21}\left\{P_{3}(x) u^{\prime}(b)+\rho P_{2}(b) u(x)\right\} \\
& \left.+\beta_{20}\left\{P_{3}(x) u(b)-P_{3}(b) u(x)\right\}\right] .
\end{aligned}
$$

If in this expression we replace each $P$ and each $u$ by its value in terms of the $\delta$ functions, the coefficients of the $\beta$ 's are each seen to equal zero. Consequently we have 
(11)

$$
\begin{aligned}
& I_{n}^{\prime}(x)= \frac{1}{2} f(x)+\epsilon_{n}(x) \\
&-\frac{1}{2 \pi i} \int_{\gamma_{n}^{\prime}}\left\{\frac{\beta_{22}\left[\rho^{2} u(x) R_{1}(b)-u^{\prime \prime}(b) R_{3}(x)\right]-\beta_{21}\left[\rho u(x) R_{2}(b)+u^{\prime}(b) R_{8}(x)\right]}{\rho^{2}\left[3 \rho^{2} D_{a}-W_{2 b}(u)\right]}\right. \\
&\left.\quad+\frac{\beta_{20}\left[u(x) R_{3}(b)-u(b) R_{3}(x)\right]}{\rho^{8}\left[3 \rho^{2} D_{a}-W_{2 b}(u)\right]}\right\} d \rho,
\end{aligned}
$$

in which $\epsilon_{n}(x)$ stands for that portion of $I_{n}^{\prime}(x)$ arising from the neglected terms.

The coefficient of each $\beta$ in the numerator of this integrand is to be considered by itself. We define variable $y$ by means of the equation

$$
y-a=(x-a) e^{i(x-\arg \rho)}
$$

In the following we also change the variables of integration by the transformations $s=a+\omega_{i}{ }^{2}(t-a)$. We have

$$
\begin{aligned}
\int_{a}^{x} \delta_{2}[\rho(x-s)] \phi_{1}^{\prime \prime}(s) d s & =\sum_{1}^{3} \omega_{i}^{2} e^{\omega_{i \rho} x} \int_{a}^{x} e^{-\omega_{i \rho s} \phi_{1}^{\prime \prime}}(s) d s \\
& =\sum_{1}^{8} e^{\omega_{i} \rho(x-a)} \int_{a}^{a-\omega_{i}(x-a)} e^{\rho(t-a)} \phi_{1}^{\prime \prime}(t) d t \\
& =\delta_{1}[\rho(x-a)] \mu_{1}+\sum_{1}^{3} e^{\omega_{i} \rho(x-a)} \nu_{i 1},
\end{aligned}
$$

where

$$
\mu_{j}=\int_{a}^{\nu} e^{p(t-a)} \phi_{j}^{(j+1)}(t) d t
$$

and

$$
\nu_{i j}=\int_{y}^{a-\omega_{i}(x-a)} e^{\rho(t-a)} \phi_{j}^{(j+1)}(t) d t .
$$

In order to render these steps and the following ones valid we require each of the functions $\phi_{j}(t)$ to be analytic in a circle centered at $t=a$. Let $r<b-a$ be the radius of this circle. We require the real number $x$ to be within this circle. Then the complex variable $y$, as well as the numbers $a-\omega_{i}(x-a)$ will be within the circle. Hence, taking the paths of $t$-integration as straight lines, these paths will lie entirely within the circle. By steps similar to those above we find

$$
\int_{a}^{x} \delta_{3}[\rho(x-s)] \phi_{2}^{\prime \prime \prime}(s) d s=\delta_{2}[\rho(x-a)] \mu_{2}+\sum_{1}^{8} \omega_{2}^{2} \nu_{i 2} e^{\omega_{i} \rho(x-a)},
$$


and

$$
\int_{a}^{x} \delta_{1}[\rho(x-s)] \phi_{3}{ }^{i v}(s) d s=\delta_{3}[\rho(x-a)] \mu_{3}-\sum_{1}^{8} \omega_{i} \nu_{i 3} e^{\omega_{i} \rho(x-a)} .
$$

From these we get

(12) $R_{3}(x)=-\rho \delta_{1}[\rho(x-a)] \mu_{1}+\delta_{2}[\rho(x-a)] \mu_{2}-\frac{1}{\rho} \delta_{3}[\rho(x-a)] \mu_{8}+A_{2}$, where

$$
A_{3}=\sum_{1}^{3} e^{\left.\omega_{i \rho} \rho-a\right)}\left[-\rho \nu_{i 1}+\omega_{i}^{2} \nu_{i 2}+\frac{1}{\rho} \omega_{i} \nu_{i 3}\right] \text {. }
$$

The reason for breaking up $R_{3}(x)$ in this way is that the portion of $R_{3}(x)$ containing the $\mu$ terms does not yield in the $\rho$-integration a quantity which has zero for its limit as $n \rightarrow \infty$, but does cancel against a similar portion of the other part of the coefficient of the $\beta$ under consideration. The $A_{3}$ portion of $R_{\mathbf{3}}(x)$ does yield in the $\rho$-integration a quantity which has zero for its limit as $n \rightarrow \infty$.

We have also

$$
\begin{aligned}
R_{8}(b)= & -\rho \int_{a}^{x} \delta_{2}[\rho(b-s)] \phi_{1}^{\prime \prime}(s) d s+\int_{a}^{x} \delta_{3}[\rho(b-s)] \phi_{2}{ }^{\prime \prime \prime}(s) d s \\
& -\frac{1}{\rho} \int_{a}^{x} \delta_{1}[\rho(b-s)] \phi_{8}{ }^{i v}(s) d s+C_{3}
\end{aligned}
$$

where

$$
\begin{aligned}
C_{3}= & -\rho \int_{x}^{b} \delta_{2}[\rho(b-s)] \phi_{1}^{\prime \prime}(s) d s+\int_{x}^{b} \delta_{3}[\rho(b-s)] \phi_{2}{ }^{\prime \prime \prime}(s) d s \\
& -\frac{1}{\rho} \int_{z}^{b} \delta_{1}[\rho(b-s)] \phi_{3}{ }^{l v}(s) d s .
\end{aligned}
$$

But

$$
\begin{aligned}
& \int_{a}^{x} \delta_{2}[\rho(b-s)] \phi_{1}^{\prime \prime}(s) d s=\delta_{1}[\rho(b-a)] \mu_{1}+\sum_{1}^{3} \nu_{i 1} e^{\omega_{i} \rho(b-a)}, \\
& \int_{a}^{x} \delta_{3}[\rho(b-s)]{\phi_{2}}^{\prime \prime \prime}(s) d s=\delta_{2}[\rho(b-a)] \mu_{2}+\sum_{1}^{3} \omega_{i}^{2} \nu_{i 2} e^{\omega_{i} \rho(b-a)}, \\
& \int_{a}^{x} \delta_{1}[\rho(b-s)] \phi^{i v}(s) d s=\delta_{3}[\rho(b-a)] \mu_{3}-\sum_{1}^{3} \omega_{i} \nu_{i 3} e^{\omega_{i} \rho(b-a)}
\end{aligned}
$$

Hence (14) becomes

(16) $R_{3}(b)=-\rho \delta_{1}[\rho(b-a)] \mu_{1}+\delta_{2}[\rho(b-a)] \mu_{2}-\frac{1}{\rho} \delta_{3}[\rho(b-a)] \mu_{3}+B_{3}+C_{3}$, 
where

$$
B_{3}=\sum_{1}^{3} e^{\omega_{i \rho}(b-a)}\left[-\rho \nu_{i 1}+\omega_{i}^{2} \nu_{i 2}+\frac{1}{\rho} \omega_{i} \nu_{i 3}\right]
$$

From (4), (12), and (16) we get for the coefficient of $\beta_{20}$ in the numerator of the integrand in (11) the expression

$$
\begin{aligned}
& \rho^{2}\left\{\delta_{1}[\rho(x-a)] \delta_{2}[\rho(b-a)]-\delta_{2}[\rho(x-a)] \delta_{1}[\rho(b-a)]\right\}\left\{\alpha_{12} \alpha_{31} \mu_{2}\right. \\
+ & \left.\alpha_{12} \alpha_{30} \mu_{1}\right\}+\rho\left\{\delta_{3}[\rho(x-a)] \delta_{1}[\rho(b-a)]-\delta_{1}[\rho(x-a)] \delta_{3}[\rho(b-a)]\right\} \\
& \cdot\left\{\alpha_{12} \alpha_{31} \mu_{3}-\left(\alpha_{11} \alpha_{30}-\alpha_{10} \alpha_{31}\right) \mu_{1}\right\}+\left\{\delta_{3}[\rho(x-a)] \delta_{2}[\rho(b-a)]\right. \\
& \left.-\delta_{2}[\rho(x-a)] \delta_{3}[\rho(b-a)]\right\}\left\{\alpha_{12} \alpha_{30} \mu_{3}+\left(\alpha_{11} \alpha_{30}-\alpha_{10} \alpha_{31}\right) \mu_{2}\right\} \\
+ & u(x)\left(B_{3}+C_{3}\right)-u(b) A_{3} .
\end{aligned}
$$

Now

$$
\alpha_{31} \mu_{2}+\alpha_{30} \mu_{1}=\int_{a}^{y} e^{p(t-a)}\left[\alpha_{31} \phi_{2}{ }^{\prime \prime}(t)+\alpha_{30} \phi_{1}^{\prime \prime}(t)\right] d t
$$

which is seen to equal zero provided $\left(8^{\prime \prime}\right)$ is satisfied. For similar reasons $\alpha_{12} \alpha_{31} \mu_{3}-\left(\alpha_{11} \alpha_{30}-\alpha_{10} \alpha_{31}\right) \mu_{1}=0$ and $\alpha_{12} \alpha_{30} \mu_{3}+\left(\alpha_{11} \alpha_{30}-\alpha_{10} \alpha_{31}\right) \mu_{2}=0$. Hence (18) reduces to $u(x)\left(B_{3}+C_{3}\right)-u(b) A_{3}$.

In exactly the same way the coefficients of $\beta_{21}$ and $\beta_{22}$ in the numerator of the integrand in (11) reduce, and we obtain

$$
\begin{array}{r}
I_{n}^{\prime}(x)=\frac{1}{2} f(x)+\epsilon_{n}(x)-\frac{1}{2 \pi i} \int_{\gamma_{n}^{\prime}}\left\{\frac{\beta_{22}\left[\rho^{2} u(x)\left(B_{1}+C_{1}\right)-u^{\prime \prime}(b) A_{3}\right]}{\rho^{2}\left[3 \rho^{2} D_{a}-W_{2 b}(u)\right]}\right. \\
\left.-\frac{\beta_{21}\left[\rho u(x)\left(B_{2}+C_{2}\right)+u^{\prime}(b) A_{3}\right]+\beta_{20}\left[u(x)\left(B_{3}+C_{3}\right)-u(b) A_{3}\right.}{\rho^{3}\left[3 \rho^{2} D_{a}-W_{2 b}(u)\right]}\right\} d \rho,
\end{array}
$$

where

$$
\begin{gathered}
B_{2}=\sum_{1}^{3} e^{\omega i \rho(b-a)}\left[\rho \omega_{i} \nu_{i 1}+\nu_{i 2}-\frac{1}{\rho} \omega_{i}^{2} \nu_{i 3}\right] \\
B_{1}=\sum_{1}^{3} e^{\omega_{i} \rho(b-a)}\left[-\rho \omega_{i}^{2} \nu_{i 1}-\omega_{i} \nu_{i 2}-\frac{1}{\rho} \nu_{i 3}\right] \\
C_{2}=-\rho \int_{x}^{b} \delta_{1}[\rho(b-s)] \phi_{1}^{\prime \prime}(s) d s+\int_{x}^{b} \delta_{2}[\rho(b-s)] \phi_{2}{ }^{\prime \prime \prime}(s) d s \\
-\frac{1}{\rho} \int_{x}^{b} \delta_{3}[\rho(b-s)] \phi_{3}{ }^{\mathrm{iv}}(s) d s, \\
C_{1}=-\rho \int_{x}^{b} \delta_{3}[\rho(b-s)] \phi_{1}{ }^{\prime \prime}(s) d s+\int_{x}^{b} \delta_{1}[\rho(b-s)] \phi_{2}{ }^{\prime \prime \prime}(s) d s \\
-\frac{1}{\rho} \int_{x}^{b} \delta_{2}[\rho(b-s)] \phi_{3}{ }^{\mathrm{iv}}(s) d s .
\end{gathered}
$$


Consider next the portions of the integral in (19) arising from the terms containing $A_{3}$. We see from (13) that $A_{3}$ itself contains nine integrals of the type

$$
\int_{y}^{a-\omega_{i}(x-a)} e^{\rho(t-a)} \phi_{j}^{(j+1)}(t) d t .
$$

In every one of these integrals $\left|e^{\rho(t-a)}\right|$ takes on its largest value along the line of integration at the upper limit. For, as $t$ runs from $y$ to $a-\omega_{i}(x-a)$, $\rho(t-a)$ runs from $-|\rho|(x-a)$ to $-\omega_{i} \rho(x-a)$. The points in the complex plane representing this latter pair of numbers are at equal distances from the origin and the former is on the negative axis of reals. Consequently the real part of $\rho(t-a)$ increases steadily as $t$ runs from the lower limit of integration to the upper. Hence, if $\left|\phi_{i}{ }^{(j+1)}(t)\right|<M$, a constant, for all $j$ entering in this discussion, we have $\left|\nu_{i j}\right|<2 M(b-a)\left|e^{-\omega_{i p}(x-a)}\right|$. Hence $\left|A_{3}\right|<K|\rho|$, where $K$ is independent of $\rho$ and of $x$. The portion of the integrand containing $A_{8}$ is

$$
\frac{W_{2 b}(u)}{3 \rho^{2} D_{a}-W_{2 b}(u)} \cdot \frac{A_{3}}{\rho^{3}} .
$$

We have seen that the first of these factors is a bounded function of $\rho$ for $|\rho|$ large and on $\gamma_{n}^{\prime}$. Hence the portion of the integrand containing $A_{3}$ can be neglected.

Consider now the remaining portion of the numerator of the integrand in (19). Formal reductions yield

$$
\begin{aligned}
u(x) & {\left[\beta_{22} \rho^{2} B_{1}-\beta_{21 \rho} B_{2}+\beta_{20} B_{3}\right] } \\
= & \sum_{i=1}^{s} e^{\omega_{i j} \rho(b-a)}\left\{\omega_{i}{ }^{2} D_{a 4} \rho^{5}-\omega_{i} D_{a 3} \rho^{4}+D_{a 2 \rho^{3}}+\omega_{i}{ }^{2} D_{a 1} \rho^{2}\right. \\
& \left.\quad-\omega_{i} D_{a 0} \rho\right\}\left\{\delta_{1}[\rho(x-a)] \nu_{i 1}-\frac{1}{\rho} \delta_{2}[\rho(x-a)] \nu_{i 2}+\frac{1}{\rho^{2}} \delta_{3}[\rho(x-a)] \nu_{i 3}\right\},
\end{aligned}
$$

and show that $u(x)\left[\beta_{22} \rho^{2} C_{1}-\beta_{21} \rho C_{2}+\beta_{20} C_{3}\right]$ equals a sum of five products of which the $i$ th is of the form

$$
\begin{aligned}
D_{a i \mathrm{i}}{ }^{i+1}\{ & \delta_{1}[\rho(x-a)] \int_{x}^{b} \delta_{j}[\rho(b-s)] \phi_{1}{ }^{\prime \prime}(s) d s \\
& \quad-\frac{1}{\rho} \delta_{2}[\rho(x-a)] \int_{x}^{b} \delta_{k}[\rho(b-s)] \phi_{2}{ }^{\prime \prime}(s) d s \\
& \left.+\frac{1}{\rho^{2}} \delta_{3}[\rho(x-a)] \int_{x}^{b} \delta_{l}[\rho(b-s)] \phi_{3}{ }^{\mathrm{iv}}(s) d s\right\},
\end{aligned}
$$


$i, k, l$ having the values $1,2,3$ in various orders in these terms. Hence

$$
\begin{aligned}
& \left|u(x)\left[\beta_{22} \rho^{2} B_{1}-\beta_{21} \rho B_{2}+\beta_{20} B_{3}\right]\right| \\
& \quad<6 M(b-a)\left|e^{\cos \rho(x-a)}\right| \sum_{i=1}^{3}\left|e^{\omega_{i} \rho(b-a)}\right| \cdot\left|D_{a j} \rho^{j+1}\right| \cdot\left|e^{-\omega_{i \rho}(x-a)}\right| \\
& \quad<K\left|\rho^{j+1} e^{\omega_{32} \rho(b-a)}\right|,
\end{aligned}
$$

where $K$ is independent of $\rho$ and of $x$. Also

$$
\left|u(x)\left[\beta_{22} \rho^{2} C_{1}-\beta_{21} \rho C_{2}+\beta_{20} C_{3}\right]\right|<K^{\prime}\left|\rho^{i+1} e^{\omega_{2} \rho(b-a)}\right|,
$$

$K^{\prime}$ being independent of $\rho$ and of $x$. Hence the portion of the integrand in (19) containing the $B$ and $C$ terms is less in numerical value than some constant divided by $\left|\rho^{2}\right|$, and can be neglected. This leaves $I_{n}^{\prime}(x)=\frac{1}{2} f(x)$ $+\epsilon_{n}(x)$, where $\epsilon_{n}(x)$ tends uniformly to zero as a limit when $n \rightarrow \infty$.

The portion of $I_{n}(x)$ arising from integration over the arc $\gamma_{n}^{\prime \prime}$ is treated in a fashion quite similar to the above treatment of $I_{n}^{\prime}(x)$ and with similar results. We omit the consideration of it, and state immediately

THEOREM 2. If the boundary conditions can be written in the form (3) with at least one $D_{a}$ not zero; if $f(x)=\phi_{1}(x)+\phi_{2}(x)+\phi_{3}(x)$, where $\phi_{1}(x)=\sum_{0}^{\infty} c_{8 n}$ - $(x-a)^{3 n}, \phi_{2}(x)=\sum_{0}^{\infty} c_{1+3 n}(x-a)^{1+3 n}, \phi_{3}(x)=\sum_{0}^{\infty} c_{2+3 n}(x-a)^{2+3 n}$ are power series convergent in and on the boundary of a circle centered at $x=a$ and of radius $r<b-a$ which satisfy the identities $\alpha_{12} \phi_{3}^{\prime \prime}(x)+\alpha_{11} \phi_{2}^{\prime}(x)+\alpha_{10} \phi_{1}(x) \equiv 0$, $\alpha_{31} \phi_{2}^{\prime}(x)+\alpha_{30} \phi_{1}(x) \equiv 0$; and if also $\phi_{1}^{\prime \prime}(x), \phi_{2}^{\prime \prime \prime}(x), \phi_{3}{ }^{\text {iv }}(x)$ are continuous for real values of $x$ from a to $b$, then the formal series for $f(x)$ of type (6) converges uniformly to $f(x)$ along the segment of the real axis from a to $a+r$.

From Theorem 1 it follows as a corollary to Theorem 2 that the formal series will converge uniformly to $f(x)$ in the equilateral triangle whose center is at $x=a$ and one vertex of which is at $x=a+r$.

We come next to the case in which two of the boundary conditions bear only at $x=b$. We do no more than state the results. Let the boundary conditions be written in the form

$$
\begin{aligned}
\beta_{12} u^{\prime \prime}(b)+\beta_{11} u^{\prime}(b)+\beta_{10} u(b) & =0, \\
\alpha_{22} u^{\prime \prime}(a)+\alpha_{21} u^{\prime}(a)+\alpha_{20} u(a)+\beta_{22} u^{\prime \prime}(b)+\beta_{21} u^{\prime}(b)+\beta_{20} u(b) & =0, \\
\beta_{23} u^{\prime}(b)+\beta_{30} u(b) & =0 .
\end{aligned}
$$

The characteristic numbers are found to lie along the rays $\arg \rho=\pi / 3$, $\pi, 5 \pi / 3$. We restrict $\rho$ to the sector $2 \pi / 3 \leqq \arg \rho \leqq 4 \pi / 3$, using the characteristic numbers along the ray $\arg \rho=\pi$. 
On the assumption of uniform convergence of $\sum_{1}^{\infty} b_{k} u_{k}(x)$ in an interval $\alpha \leqq x \leqq \beta$, where $a<\alpha<\beta<b$, we obtain a restriction on the $b_{k}$ 's which is of sufficient strength to allow us to show that the series converges uniformly in the equilateral triangle centered at $x=b$ and having one vertex at $x=\alpha$. A formal property of the characteristic functions then shows that the series necessarily represents a function of the form $f(x)=\phi_{1}(x)+\phi_{2}(x)+\phi_{3}(x)$, where

$$
\begin{aligned}
& \phi_{1}(x)=\sum_{0}^{\infty} c_{3 n}(x-b)^{3 n}, \quad \phi_{2}(x)=\sum_{0}^{\infty} c_{1+3 n}(x-b)^{1+8 n}, \\
& \phi_{3}(x)=\sum_{0}^{\infty} c_{2+3 n}(x-b)^{2+3 n},
\end{aligned}
$$

each $\phi$ being analytic in the triangle, and that the $\phi$ 's must satisfy the identities

$$
\beta_{12} \phi_{3}^{\prime \prime}(x)+\beta_{11} \phi_{2}^{\prime}(x)+\beta_{10} \phi_{1}(x) \equiv 0, \quad \beta_{31} \phi_{2}^{\prime}(x)+\beta_{80} \phi_{1}(x) \equiv 0 .
$$

If we now take a function $f(x)$ satisfying the above conditions and in addition such that $\phi_{1}^{\prime \prime}(x), \phi_{2}^{\prime \prime \prime}(x)$, and $\phi_{3}^{\text {iv }}(x)$ are continuous from $a$ to $b$, the formal series for $f(x)$ of type $\sum_{1}^{\infty} b_{k} u_{k}(x)$ will converge uniformly to $f(x)$ in the interval $(b-r, b), r$ being the radius of the circle in which the $\phi$ 's are assumed to be analytic.

\section{PART II}

We have made reference to a very special case treated by Hopkins in which the boundary conditions can be written $u(0)=u^{\prime}(0)=u(\pi)=0$. The characteristic functions in this case are $u_{k}(x)=\delta_{3}\left(\rho_{k} x\right), k=1,2, \cdots$, and they satisfy the functional equations $u_{k}\left(-\omega_{2} x\right)=-\omega_{3} u_{k}(x), u_{k}\left(-\omega_{3} x\right)$ $=-\omega_{2} u_{k}(x)$. Hence $u_{k}\left(-\omega_{2} \pi\right)=u_{k}\left(-\omega_{3} \pi\right)=0$. Also, denoting differentiations with respect to $x$ by accents, $u_{k}^{\prime \prime}\left(-\omega_{3} x\right)=u_{k}^{\prime \prime}(x)$. Hence $u_{k}^{\prime \prime}\left(-\omega_{3} \pi\right)=0$. From these we select

$$
u(\pi)=u\left(-\omega_{3} \pi\right)=0, \quad u^{\prime \prime}(\pi)=u^{\prime \prime}\left(-\omega_{3} \pi\right),
$$

which we shall take as boundary conditions. They will be seen to lead to a larger class of characteristic functions than Hopkins', but one which includes his.

Use of the first two of (20) gives

$$
u(x)=\left(e^{2 \omega_{2} \rho \pi}-e^{-\omega_{2} \rho \pi}\right) e^{\omega_{1} \rho x}+\left(e^{2 \omega_{3} \rho \pi}-e^{-\omega_{3} \rho \pi}\right) e^{\omega_{2} \rho x}+\left(e^{2 \omega_{1} \rho \pi}-e^{-\omega_{1} \rho \pi}\right) e^{\omega_{3} \rho x},
$$

at least to a factor independent of $x$. The characteristic equation is 


$$
\Delta(\rho) \equiv\left|\begin{array}{ccc}
e^{\omega_{1} \rho \pi} & e^{\omega_{2} \rho \pi} & e^{\omega_{2} \rho \pi} \\
e^{\omega_{2} \rho \pi} & e^{\omega_{1} \rho \pi} & e^{\omega_{2} \rho \pi} \\
\omega_{1}^{2} \rho^{2}\left(e^{\omega_{1} \rho \pi}-e^{\omega_{3} \rho \pi}\right) & \omega_{2}^{2} \rho^{2}\left(e^{\omega_{2} \rho \pi}-e^{\omega_{1} \rho \pi}\right) & \omega_{3}^{2} \rho^{2}\left(e^{\omega_{2} \rho \pi}-e^{\omega_{2} \rho \pi}\right)
\end{array}\right|
$$

The fact that the characteristic equation factors in the particular way indicated here is what suggested the choice of boundary conditions under consideration. This choice, of course, is only one of many that might be made. The factor $\delta_{3}(\rho \pi)$ of (22) has for its zeros precisely Hopkins' characteristic numbers, which we denote by $\rho_{1 k}$. The other factor $\delta_{3}(-\rho \pi)$ has the negatives of the numbers $\rho_{1 k}$ for its zeros; we denote these by $\rho_{2 k}$, and write $\rho_{2 k}=-\rho_{1 k}$.

We have

and

$$
e^{\omega_{1} \rho_{1 k} \pi}-\omega_{2} e^{\omega_{2} \rho_{1 k} \pi}-\omega_{3} e^{\omega_{3} \rho_{1} \pi}=0
$$

$$
e^{\omega_{3} \rho_{1} k^{\pi}}-\omega_{2} e^{\omega_{1} \rho_{1 k} \pi}-\omega_{3} e^{\omega_{2} \rho_{1 k} \pi}=0,
$$

the first being Hopkins' characteristic equation, and the second being obtained by multiplying the first by $-\omega_{2}$ and rearranging the terms. From these two equations we get

$$
1:-\omega_{2}:-\omega_{3}=e^{2 \omega_{2} \rho_{1 k} \pi}-e^{-\omega_{2} \rho_{1 k} \pi}: e^{2 \omega_{3} \rho_{1 k} \pi}-e^{-\omega_{8} \rho_{1 k} \pi}: e^{2 \omega_{1} \rho_{1 k} \pi}-e^{-\omega_{1} \rho_{1 k} \pi} .
$$

Consequently if in (21) we replace $\rho$ by $\rho_{1 k}$ and remove the proper factor independent of $x$, we get what we shall take as one set of characteristic functions, namely $u_{1 k}(x)=\delta_{3}\left(\rho_{1 k} x\right)$. These are Hopkins' characteristic functions. If we transform by means of the transformation

$$
x^{\prime}=\omega_{2} \pi-x
$$

to the point $\omega_{2} \pi$ as a new origin, we find by steps similar to those above that the rest of our characteristic functions are given by $u_{2 k}(x)=\delta_{3}\left(\rho_{1 k} x^{\prime}\right)=$ $\delta_{3}\left[\rho_{1 k}\left(\omega_{2} \pi-x\right)\right]$.

From Part I we know that if the series

$$
\sum_{1}^{\infty} a_{1 k} u_{1 k}(x)
$$

converges uniformly in some segment of the axis of reals from 0 to $\pi$, it represents a function analytic in $x$ and of the form $x^{2} \phi\left(x^{3}\right), \phi\left(x^{3}\right)$ being a convergent power series in $x^{3}$, and that the series also converges uniformly in an equilateral triangle centered at $x=0$ and having one vertex on the axis of reals between 0 and $\pi$. Noting that the transformation (23) consists of 
a parallel shift $x^{\prime \prime}=x-\omega_{2} \pi$ followed by a rotation through $180^{\circ}, x^{\prime}=-x^{\prime \prime}$, we see that if a series

$$
\sum_{1}^{\infty} a_{2 k} u_{2 k}(x)
$$

converges uniformly in some segment of the straight line between $\omega_{2} \pi$ and $-\omega_{3} \pi$, it represents a function of the form $\left(\omega_{2} \pi-x\right)^{2} \psi\left[\left(\omega_{2} \pi-x\right)^{3}\right]$, where $\psi\left[\left(\omega_{2} \pi-x\right)^{3}\right]$ is a convergent power series in $\left(\omega_{2} \pi-x\right)^{3}$, and the series also converges uniformly in an equilateral triangle centered at $x=\omega_{2} \pi$ and having one vertex on the segment from $\omega_{2} \pi$ to $-\omega_{3} \pi$. If the triangle centered at $x=0$, which we call triangle $I$, and the triangle centered at $x=\omega_{2} \pi$, which we call triangle II, are so large as to have one side in common, it is conceivable that on this common side a series of the type

$$
\sum_{1}^{\infty}\left[a_{1 k} u_{1 k}(x)+a_{2 k} u_{2 k}(x)\right]
$$

may represent functions of a wider class than those represented by either series (24) or (25) individually or than a linear combination of functions representable by series (24) and (25).

Let, therefore, triangles I and II be so large that they have a common side $C$, which will be the segment from $\pi$ to $-\omega_{3} \pi$. Putting $f(x)$ equal to series (26), how shall we determine formally the $a$ 's? To answer this question we proceed to a definition of adjoints.

Write $L(u)=u^{\prime \prime \prime}+\rho^{3} u$ and $M(v)=-v^{\prime \prime \prime}+\rho^{3} v$. Then

$$
\int_{C}[v L(u)-u M(v)] d x=\int_{C}\left[v u^{\prime \prime \prime}-u v^{\prime \prime \prime}\right] d x=\left[u v^{\prime \prime}-u^{\prime} v^{\prime}+u^{\prime \prime} v\right]_{C} .
$$

If we require that $u(\pi)=u\left(-\omega_{3} \pi\right)=0, u^{\prime \prime}(\pi)=u^{\prime \prime}\left(-\omega_{3} \pi\right)$, and $v^{\prime}(\pi)=$ $v^{\prime}\left(-\omega_{3} \pi\right)=0, v(\pi)=v\left(-\omega_{3} \pi\right)$, we have

$$
\int_{C}[v L(u)-u M(v)] d x=0 .
$$

If we require also that $u$ satisfy $L(u)=0, u$ will be a characteristic function $u_{1 i}(x)$ or $u_{2 i}(x)$. The functions $v$ satisfying the above conditions at $\pi$ and $-\omega_{3} \pi$ and also the differential equation $M(v)=0$ we define as the adjoints of the $u$ characteristic functions. Mere formal manipulations suffice to show that the characteristic numbers for the $v$ differential system are the same as those for the $u$ system. Adopting an obvious notation for the $v$ characteristic functions, we have 


$$
\int_{C}\left[v_{2 j} L\left(u_{1 i}\right)-u_{1 i} M\left(v_{2 j}\right)\right] d x=0 .
$$

But

$$
\int_{C}\left[v_{2 j} L\left(u_{1 i}\right)-u_{1 i} M\left(v_{2 j}\right)\right] d x=\left(\rho_{1 i}^{3}-\rho_{2 j}^{3}\right) \int_{0} u_{1 i} v_{2 j} d x
$$

Hence

$$
\begin{array}{ll}
\int_{C} u_{1 ;} v_{2 j} d x=0 & \text { for all } i \text { and } j ; \text { and similarly } \\
\int_{C} u_{2 i} v_{1 j} d x=0 & \text { for all } i \text { and } j, \\
\int_{C} u_{1 i} v_{1 j} d x=0 & \text { if } i \neq j, \text { and } \\
\int_{C} u_{2 i} v_{2 j} d x=0 & \text { if } i \neq j .
\end{array}
$$

All integrations over $C$ are to be made from $-\omega_{3} \pi$ to $\pi$. The functions $v$ are found to be given by $v_{1 k}(x)=\delta_{1}\left[\rho_{1 k}\left(\omega_{2} \pi-x\right)\right]$ and $\nu_{2 k}(x)=\delta_{1}\left(\rho_{1 k} x\right)$, except possibly for factors independent of $x$, of which we take no account. The relations (27) enable us to determine formally values for the $a$ 's in series (26).

We proceed to study series (26) under the hypothesis that it converges uniformly in some segment of $C$. Writing $x=\xi+(\pi-\xi) i / 3^{1 / 2}$, which yields $x$ on the line of $C$ if $\xi$ is real, we have

$$
\begin{aligned}
u_{1 k}(x)= & e^{-\rho_{k} \xi}\left[\cos \rho_{k} \frac{\pi-\xi}{3^{1 / 2}}-i \sin \rho_{k} \frac{\pi-\xi}{3^{1 / 2}}\right] \\
-e^{\rho k(\xi-\pi / 2)} & {\left[\cos \left(\frac{\pi}{3}+\frac{\rho_{k} \xi}{3^{1 / 2}}+\frac{\rho_{k} \pi}{2 \cdot 3^{1 / 2}}\right)\right.} \\
& \left.+i \sin \left(\frac{\pi}{3}+\frac{\rho_{k} \xi}{3^{1 / 2}}+\frac{\rho_{k} \pi}{2 \cdot 3^{1 / 2}}\right)\right] \\
& -e^{\rho_{k} \pi / 2}\left[\cos \left(\frac{\pi}{3}+\frac{2 \rho_{k} \xi}{3^{1 / 2}}+\frac{\rho_{k} \pi}{2 \cdot 3^{1 / 2}}\right)\right. \\
& \left.-i \sin \left(\frac{\pi}{3}+\frac{2 \rho_{k} \xi}{3^{1 / 2}}-\frac{\rho_{k} \pi}{2 \cdot 3^{1 / 2}}\right)\right]
\end{aligned}
$$


and

$$
\begin{aligned}
u_{2 k}(x)= & e^{\rho_{k}(\xi-x / 2)}\left[\cos \left(\frac{\rho_{k} \pi}{2 \cdot 3^{1 / 2}}+\frac{\rho_{k} \xi}{3^{1 / 2}}\right)-i \sin \left(\frac{\rho_{k} \pi}{2 \cdot 3^{1 / 2}}+\frac{\rho_{k} \xi}{3^{1 / 2}}\right)\right] \\
& -e^{-\rho_{k} \xi}\left[\cos \left(\frac{\pi}{3}+\frac{\rho_{k} \pi}{3^{1 / 2}}-\frac{\rho_{k} \xi}{3^{1 / 2}}\right)+i \sin \left(\frac{\pi}{3}+\frac{\rho_{k} \pi}{3^{1 / 2}}-\frac{\rho_{k} \xi}{3^{1 / 2}}\right)\right] \\
-e^{\rho_{k} \pi / 2} & {\left[\cos \left(\frac{\pi}{3}+\frac{\rho_{k} \pi}{2 \cdot 3^{1 / 2}}-\frac{2 \rho_{k} \xi}{3^{1 / 2}}\right)-i \sin \left(\frac{\pi}{3}+\frac{\rho_{k} \pi}{2 \cdot 3^{1 / 2}}-\frac{2 \rho_{k} \xi}{3^{1 / 2}}\right)\right] . }
\end{aligned}
$$

Here we have used $\rho_{k}$ to stand for $\rho_{1 k}$, and we suppose not only that $-\pi / 2<\xi<\pi$, so that $x$ will be on $C$, but also that $\xi$ is so restricted that $x$ is within the range of assumed uniform convergence of (26).

Take $k$ so large that there will be a value $\xi^{\prime}$ of $\xi$ in its restricted range such that

$$
\cos \left(\frac{\pi}{3}+\frac{\rho_{k} \pi}{2 \cdot 3^{1 / 2}}-\frac{2 \rho_{k} \xi}{3^{1 / 2}}\right)=1 .
$$

If $x^{\prime}$ is the value of $x$ which corresponds to $\xi^{\prime}$, we find

$$
u_{1 k}\left(x^{\prime}\right)=e^{\rho_{k \pi / 2}}\left[\omega_{2}+E_{1 k}\right] \text { and } u_{2 k}\left(x^{\prime}\right)=e^{\rho k \pi / 2}\left[-1+E_{2 k}\right] \text {, }
$$

where $E_{1 k}$ and $E_{2 k}$ both can be made as small numerically as desired by taking $k$ sufficiently large. Consequently

$$
a_{1 k}\left(\omega_{2}+E_{1 k}\right)+a_{2 k}\left(-1+E_{2 k}\right)=e^{-p k \pi / 2}\left[a_{1 k} u_{1 k}\left(x^{\prime}\right)+a_{2 k} u_{2 k}\left(x^{\prime}\right)\right]
$$

If $k$ is large enough, we can repeat the work of this paragraph with another value $\xi^{\prime \prime}$ of $\xi$, such that

$$
\cos \left(\frac{\pi}{3}+\frac{2 \rho_{k} \xi^{\prime \prime}}{3^{1 / 2}}-\frac{\rho_{k} \pi}{2 \cdot 3^{1 / 2}}\right)=1,
$$

and if $x^{\prime \prime}$ is the corresponding value of $x$, we shall obtain

$$
a_{1 k}\left(-1+E_{1 k}^{1}\right)+a_{2 k}\left(\omega_{2}+E_{2 k}^{1}\right)=e^{-p k \pi / 2}\left[a_{1 k} u_{1 k}\left(x^{\prime \prime}\right)+a_{2 k} u_{2 k}\left(x^{\prime \prime}\right)\right] .
$$

Equations (30) and $\left(30^{\prime}\right)$ are to be solved as non-homogeneous linear equations for the $a_{1 k}$ and $a_{2 k}$ which appear in their left hand members. If the solution is made in the usual way by determinants, we find $a_{1 k}$ given by a quotient of two determinants times $e^{-\rho_{k} \pi / 2}$. The determinant in the denominator of this quotient is seen to have a numerical value greater than some positive constant, while the determinant in the numerator is less numerically than some positive constant, since $\left|a_{1 k} u_{1 k}(x)+a_{2 k} u_{2 k}(x)\right|<g$, where $g$ is independent of both $x$ and $k$, holds for both $x=x^{\prime}$ and $x=x^{\prime \prime}$. Similar remarks are valid for the solution for $a_{2 k}$. Thus we have proved the 
LеммA. If series (26) converges uniformly in some segment of $C$, then $\left|a_{1 k}\right|<h_{1} e^{-\rho \alpha^{*} / 2}$ and $\left|a_{2 k}\right|<h_{2}^{-\rho \mu^{*} / 2}, h_{1}$ and $h_{2}$ being independent of $k$.

From this lemma we may prove the following theorem by the method of reasoning used in the work immediately following the lemma of Part I.

THEOREM 1. If series (26) converges uniformly in some segment of $C$, then series (24) converges uniformly in the interior of triangle I and represents a function analytic in $x$ and of the form $x^{2} \phi\left(x^{3}\right)$, and series (25) converges uniformly in the interior of triangle II and represents a function analytic in $x$ and of the form $\left(\omega_{2} \pi-x\right)^{2} \psi\left[\left(\omega_{2} \pi-x\right)^{3}\right]$.

Let us now write purely formally $f(x)=\sum_{1}^{\infty}\left[a_{1 k} u_{1 k}(x)+a_{2 k} u_{2 k}(x)\right]$, and determine the $a$ 's by means of relations (27). We shall suppose that $f(x)$ is continuous together with its first two derivatives on the closed segment C. We find

$$
\int_{C} f(x) v_{1 k}(x) d x=a_{1 k} \int_{C} u_{1 k}(x) v_{1 k}(x) d x
$$

and

$$
\int_{C} f(x) v_{2 k}(x) d x=a_{2 k} \int_{C} u_{2 k}(x) v_{2 k}(x) d x
$$

Carrying out the integrations appearing on the right hand sides of these equations explicitly, we find

$$
\int_{C} u_{1 k}(x) v_{1 k}(x) d x=\int_{C} u_{2 k}(x) v_{2 k}(x) d x=\left(\omega_{2}-\omega_{3}\right) \pi \delta_{3}\left(-\rho_{k} \pi\right) .
$$

Also, making two integrations by parts,

$$
\begin{aligned}
\int_{C} f(x) v_{1 k}(x) d x= & -\frac{1}{\rho_{k}}\left[\omega_{3} f(\pi)+f\left(-\omega_{3} \pi\right)\right] \delta_{2}\left(\rho_{k} \pi\right) \\
& +\frac{1}{\rho_{k}^{2}} \int_{C} \delta_{3}\left[\rho_{k}\left(\omega_{2} \pi-x\right)\right] f^{\prime \prime}(x) d x
\end{aligned}
$$

and

$$
\begin{aligned}
\int_{C} f(x) v_{2 k}(x) d x=-\frac{1}{\rho_{k}}[f(\pi)+ & \left.\omega_{3} f\left(-\omega_{3} \pi\right)\right] \delta_{2}\left(\rho_{k} \pi\right) \\
& +\frac{1}{\rho_{k}^{2}} \int_{C} \delta_{3}\left(\rho_{k} x\right) f^{\prime \prime}(x) d x
\end{aligned}
$$

We may, therefore, write 


$$
\begin{aligned}
a_{1 k}= & \frac{\omega_{3} f(\pi)+f\left(-\omega_{3} \pi\right)}{\left(\omega_{3}-\omega_{2}\right) \pi} \cdot \frac{\delta_{2}\left(\rho_{k} \pi\right)}{\rho_{k} \delta_{3}\left(-\rho_{k} \pi\right)} \\
& \quad+\frac{1}{\left(\omega_{2}-\omega_{3}\right) \pi \rho_{k}^{2} \delta_{3}\left(-\rho_{k} \pi\right)} \int_{C} \delta_{3}\left[\rho_{k}\left(\omega_{2} \pi-x\right)\right] f^{\prime \prime}(x) d x,
\end{aligned}
$$

and

$$
\begin{aligned}
a_{2 k}=\frac{f(\pi)+\omega_{3} f\left(-\omega_{3} \pi\right)}{\left(\omega_{3}-\omega_{2}\right) \pi} \cdot \frac{\delta_{2}\left(\rho_{k} \pi\right)}{\rho_{k} \delta_{3}\left(-\rho_{k} \pi\right)} \\
\quad+\frac{1}{\left(\omega_{2}-\omega_{3}\right) \pi \rho_{k}^{2} \delta_{3}\left(-\rho_{k} \pi\right)} \int_{C} \delta_{3}\left(\rho_{k} x\right) f^{\prime \prime}(x) d x .
\end{aligned}
$$

We now insert these expressions for the $a$ 's into series (24) and (25), and test these for convergence on $C$. Before actually doing this, however, we investigate the sizes of $a_{1 k}$ and $a_{2 k}$. Consider the integral in (31'). It contains in its integrand the sum of three exponentials, no one of which at any point of $C$ exceeds $e^{\rho k \pi / 2}$ in numerical value. Hence

$$
\left|\int_{C} \delta_{3}\left[\rho_{k}\left(\omega_{2} \pi-x\right)\right] f^{\prime \prime}(x) d x\right|<M e^{\rho_{k \pi / 2}},
$$

$M$ being independent of $k$. Also

$$
\frac{1}{\delta_{3}\left(-\rho_{k} \pi\right)}=e^{-\rho_{k} \pi}\left[1+\epsilon\left(\rho_{k}\right)\right]
$$

where $\epsilon\left(\rho_{k}\right)$ has the limit zero as $k \rightarrow \infty$. Hence the term of $\left(31^{\prime}\right)$ which contains the $C$ integral can be written $\left(1 / \rho_{k}^{2}\right) e^{-\rho_{k} / 2} M\left(\rho_{k}\right)$, where $M\left(\rho_{k}\right)$ is bounded for $k$ large. Now

$$
\begin{aligned}
\frac{\delta_{2}\left(\rho_{k} \pi\right)}{\delta_{3}\left(-\rho_{k} \pi\right)}= & e^{-\rho_{k} \pi / 2}\left[-2 \cos \left(\frac{\rho_{k} \pi \cdot 3^{1 / 2}}{2}-\frac{\pi}{3}\right)\right. \\
& \left.+\frac{\omega_{3} e^{-2 \omega_{2} \rho_{k} \pi+\rho_{k} \pi / 2}+\omega_{2} e^{-2 \omega_{3} \rho_{k} \pi+\rho_{k} \pi / 2}-e^{-\rho_{k} \pi / 2}}{\delta_{3}\left(-\rho_{k} \pi\right)}\right]
\end{aligned}
$$

Hence $\left(31^{\prime}\right)$ can be written

$$
a_{1 k}=e^{-\rho_{k} \pi / 2}\left[2 \frac{\omega_{3} f(\pi)+f\left(-\omega_{3} \pi\right)}{\left(\omega_{2}-\omega_{3}\right) \pi \rho_{k}} \cos \left(\frac{\rho_{k} \pi \cdot 3^{1 / 2}}{2}-\frac{\pi}{3}\right)+\frac{M^{\prime}\left(\rho_{k}\right)}{\rho_{k}^{2}}\right],
$$

where $M^{\prime}\left(\rho_{k}\right)$ is bounded for $k$ large. 
But from (28) we have on $C$

$$
\begin{aligned}
& \mu_{1 k}(x)=e^{\rho_{k} \pi / 2}\left[-\cos \left(\frac{\pi}{3}+\frac{2 \rho_{k} \xi}{3^{1 / 2}}-\frac{\rho_{k} \pi}{2 \cdot 3^{1 / 2}}\right)\right. \\
& \left.\quad+i \sin \left(\frac{\pi}{3}+\frac{2 \rho_{k} \xi}{3^{1 / 2}}-\frac{\rho_{k} \pi}{2 \cdot 3^{1 / 2}}\right)+\epsilon_{k}(\xi)\right],
\end{aligned}
$$

where $\epsilon_{k}(\xi)$ tends to zero uniformly as an exponential function when $k \rightarrow \infty$ if $x$ is restricted to a closed interval on $C$ which does not include the end points of $C$. From (35) and (36) it is clear that series (24) will converge uniformly on those segments of $C$ on which

$$
\begin{aligned}
& \sum_{1}^{\infty} \frac{1}{\rho_{k}} \cos \left(\frac{\rho_{k} \pi \cdot 3^{1 / 2}}{2}-\frac{\pi}{3}\right) \cos \left(\frac{\pi}{3}+\frac{2 \rho_{k} \xi}{3^{1 / 2}}-\frac{\rho_{k} \pi}{2 \cdot 3^{1 / 2}}\right) \text { and } \\
& \sum_{1}^{\infty} \frac{1}{\rho_{k}} \cos \left(\frac{\rho_{k} \pi \cdot 3^{1 / 2}}{2}-\frac{\pi}{3}\right) \sin \left(\frac{\pi}{3}+\frac{2 \rho_{k} \xi}{3^{1 / 2}}-\frac{\rho_{k} \pi}{2 \cdot 3^{1 / 2}}\right)
\end{aligned}
$$

both converge uniformly. From the equation whose roots are $\rho_{k}$ we get

$$
\cos \left(\frac{\rho_{k} \pi \cdot 3^{1 / 2}}{2}-\frac{\pi}{3}\right)=(-1)^{k} \frac{3^{1 / 2}}{2}\left(1-\frac{1}{4} e^{-3 \rho_{k} \pi}\right)^{1 / 2}-\frac{1}{4} e^{-3 \rho_{k} \pi / 2} .
$$

It is clear, therefore, that both series (37) will converge uniformly on those segments of $C$ on which

$$
\sum_{1}^{\infty} \frac{(-1)^{k}}{\rho_{k}} \cos \left(\frac{2 \rho_{k} \xi}{3^{1 / 2}}-\frac{\rho_{k} \pi}{2 \cdot 3^{1 / 2}}\right) \text { and } \sum_{1}^{\infty} \frac{(-1)^{k}}{\rho_{k}} \sin \left(\frac{2 \rho_{k} \xi}{3^{1 / 2}}-\frac{\rho_{k} \pi}{2 \cdot 3^{1 / 2}}\right)
$$

both converge uniformly. Since $\rho_{k}=\left(1 / 3^{1 / 2}\right)(1 / 3+2 k)+\epsilon_{k}$, where $\epsilon_{k}$ is given by

$$
\sin \frac{\pi \epsilon_{k} \cdot 3^{1 / 2}}{2}=\frac{(-1)^{k+1}}{2} e^{-3 \rho_{k} \pi / 2},
$$

we see by simple reductions that these series will converge uniformly on those segments of $C$ on which

$$
\sum_{1}^{\infty} \frac{(-1)^{k}}{\rho_{k}} \cos k s \text { and } \sum_{1}^{\infty} \frac{(-1)^{k}}{\rho_{k}} \sin k s, \quad s=\frac{1}{3}(4 \xi-\pi),
$$

both converge uniformly. However, these series are known to converge uniformly except possibly in neighborhoods of the points $s=2 l \pi$ and $s=(2 l-1) \pi .^{*}$ These exceptional points correspond on $C$ only to the end

* Bôcher, Introduction to the theory of Fourier series, Annals of Mathematics, (2), vol. 7 (1906), p. 111. 
and mid-points. Similar considerations are valid for $a_{2 k}$ and $u_{2 k}(x)$ on $C$, the work being based on (32') and (29). We may, therefore, state

THEOREM 2. If $f(x)$ is continuous together with its first two derivatives, and if $a_{1 k}$ and $a_{2 k}$ are given by (31') and (32'), then series (24), (25), and also (26) converge uniformly in all closed intervals of $C$ not containing the end or midpoints of $C$.

If series (26) converges uniformly on $C$, and $f(x)$, the function to which it converges, has a continuous second derivative on $C$, then the determinations $\left(31^{\prime}\right)$ and $\left(32^{\prime}\right)$ of $a_{1 k}$ and $a_{2 k}$ will be valid. Hence series (24) and (25) will both converge uniformly in all closed intervals of $C$ not containing the end or mid-points of $C$, and will represent on these intervals continuous functions of $x$. Referring to Theorem 1 , we see that we may now state

THEOREM 3. If series (26) converges uniformly on $C$ to a function which has a continuous second derivative on $C$, then series (24) and (25) both converge uniformly in all closed intervals of $C$ not containing the end or mid-points of $C$, series (24) converges uniformly in triangle I to an analytic function of $x$ of the form $x^{2} \phi\left(x^{3}\right)$, and series (25) converges uniformly in triangle II to an analytic function of $x$ of the form $\left(\omega_{2} \pi-x\right)^{2} \psi\left[\left(\omega_{2} \pi-x\right)^{3}\right]$.

We see, therefore, that under the hypotheses of this theorem, series (26) must represent on $C$ a function which is capable of being written in the form

$$
x^{2} \phi\left(x^{3}\right)+\left(\omega_{2} \pi-x\right)^{2} \psi\left[\left(\omega_{2} \pi-x\right)^{3}\right]
$$

on $C$, where $\phi\left(x^{3}\right)$ is an analytic function of $x$ in triangle $\mathrm{I}$ and $\psi\left[\left(\omega_{2} \pi-x\right)^{3}\right]$ an analytic function of $x$ in triangle II, both being of the forms indicated.

Let us now begin with a function $f(x)$ of the form (38) on $C$ and such that $\phi\left(x^{8}\right)$ and $\psi\left[\left(\omega_{2} \pi-x\right)^{3}\right]$ both have continuous second derivatives on $C$. Will the series (26) with coefficients given by (31) and (32) converge to $f(x)$ on $C$ ? Let us write $x^{2} \phi\left(x^{3}\right)=\sum_{1}^{\infty} a_{1 k} u_{1 k}(x)$. It is known that if

$$
a_{1 k}=\int_{0}^{\pi} x^{2} \phi\left(x^{3}\right) \delta_{3}\left[\rho_{k}(\pi-x)\right] d x \div \int_{0}^{\pi} u_{1 k}(x) \delta_{3}\left[\rho_{k}(\pi-x)\right] d x,
$$

then this series will converge uniformly to $x^{2} \phi\left(x^{3}\right)$ along a certain segment of the real axis between 0 and $\pi{ }^{*}$ We shall prove that $a_{1 k}$ thus given equals $a_{1 k}$ as given by (31). To this end we need only prove that

* Hopkins, loc. cit. 


$$
\begin{aligned}
\int_{C} f(x) v_{1 k}(x) d x & \int_{0}^{\pi} u_{1 k}(x) \delta_{3}\left[\rho_{k}(\pi-x)\right] d x \\
& =\int_{C} u_{1 k}(x) v_{1 k}(x) d x \int_{0}^{\pi} x^{2} \phi\left(x^{3}\right) \delta_{3}\left[\rho_{k}(\pi-x)\right] d x .
\end{aligned}
$$

But

$$
\int_{0}^{\pi} u_{1 k}(x) \delta_{3}\left[\rho_{k}(\pi-x)\right] d x=\pi \delta_{2}\left(\rho_{k} \pi\right) .
$$

Hence, in view of (33), we need only prove

$$
\delta_{2}\left(\rho_{k} \pi\right) \int_{C} f(x) v_{1 k}(x) d x=\left(\omega_{2}-\omega_{3}\right) \delta_{3}\left(-\rho_{k} \pi\right) \int_{0}^{\pi} x^{2} \phi\left(x^{3}\right) \delta_{3}\left[\rho_{k}(\pi-x)\right] d x .
$$

But

$$
\begin{aligned}
\int_{C} f(x) v_{1 k}(x) d x & =\int_{C}\left\{x^{2} \phi\left(x^{3}\right)+\left(\omega_{2} \pi-x\right)^{2} \psi\left[\left(\omega_{2} \pi-x\right)^{3}\right]\right\} \delta_{1}\left[\rho_{k}\left(\omega_{2} \pi-x\right)\right] d x \\
& =\int_{C} x^{2} \phi\left(x^{3}\right) \delta_{1}\left[\rho_{k}\left(\omega_{2} \pi-x\right)\right] d x,
\end{aligned}
$$

since $\left(\omega_{2} \pi-x\right)^{2} \psi\left[\left(\omega_{2} \pi-x\right)^{3}\right] \delta_{1}\left[\rho_{k}\left(\omega_{2} \pi-x\right)\right] d x$ is the differential of a singlevalued function of $\left(\omega_{2} \pi-x\right)^{3}$. Also

$$
\begin{gathered}
\int_{C} x^{2} \phi\left(x^{3}\right) \delta_{1}\left[\rho_{k}\left(\omega_{2} \pi-x\right)\right] d x=\left[\int_{-\omega_{2} \pi}^{0}+\int_{0}^{\pi}\right] x^{2} \phi\left(x^{3}\right) \delta_{1}\left[\rho_{k}\left(\omega_{2} \pi-x\right)\right] d x \\
=\int_{0}^{\pi} x^{2} \phi\left(x^{3}\right)\left\{\delta_{1}\left[\rho_{k}\left(\omega_{2} \pi-x\right)\right]-\delta_{1}\left[\rho_{k}\left(\omega_{2} \pi+\omega_{3} x\right)\right]\right\} d x
\end{gathered}
$$

by Cauchy's integral theorem together with a change of integration variable. Hence to establish (39) we need merely prove

$$
\begin{array}{r}
\delta_{2}\left(\rho_{k} \pi\right)\left\{\delta_{1}\left[\rho_{k}\left(\omega_{2} \pi-x\right)\right]-\delta_{1}\left[\rho_{k}\left(\omega_{2} \pi+\omega_{3} x\right)\right]\right\} \\
=\left(\omega_{2}-\omega_{3}\right) \delta_{3}\left(-\rho_{k} \pi\right) \delta_{3}\left[\rho_{k}(\pi-x)\right],
\end{array}
$$

the truth of which is readily established by use of the equation defining the numbers $\rho_{k}$.

But with $a_{1 k}$ as given by (31) we know that series (24) converges uniformly along $C$ except possibly at the end and mid-points. In view of the above it follows that (24) converges uniformly to $x^{2} \phi\left(x^{3}\right)$ on $C$ except possibly near the end and mid-points. In a similar way we may show that with $a_{2 k}$ as given by (32), series (25) will converge uniformly to $\left(\omega_{2} \pi-x\right)^{2} \psi\left[\left(\omega_{2} \pi-x\right)^{3}\right]$ on $C$ except possibly near the end and mid-points. We may, therefore, state 
Theorem 4. If $f(x)=x^{2} \phi\left(x^{3}\right)+\left(\omega_{2} \pi-x\right)^{2} \psi\left[\left(\omega_{2} \pi-x\right)^{3}\right]$ on $C$, where $\phi\left(x^{8}\right)$ is continuous together with its first two derivatives on $C$ and analytic in $x$ and of the form indicated in triangle $\mathrm{I}$, and where $\psi\left[\left(\omega_{2} \pi-x\right)^{3}\right]$ is continuous together with its first two derivatives on $C$ and analytic in $x$ and of the form indicated in triangle II, then the series (26) with the formal determinations of the coefficients will converge uniformly to $f(x)$ on all closed intervals of $C$ not containing an end or mid-point of $C$.

\section{PART III}

The type of expansion considered in Part II is one of a class in which the characteristic functions are determined by the differential equation $u^{\prime \prime}+\rho^{8} u=0$ and three boundary conditions imposed at two of the points $\pi,-\omega_{2} \pi$, $-\omega_{3} \pi$. We shall not consider this type further, but take instead a case in which the boundary conditions bear in a symmetric fashion at these three points. We take $u(\pi)=u\left(-\omega_{2} \pi\right)=u\left(-\omega_{3} \pi\right)=0$.

Here the characteristic equation is

$$
\Delta(\rho) \equiv\left|\begin{array}{lll}
e^{\omega_{1} \rho \pi} & e^{\omega_{2} \rho \pi} & e^{\omega_{2} \rho \pi} \\
e^{\omega_{2} \rho \pi} & e^{\omega_{2} \rho \pi} & e^{\omega_{1} \rho \pi} \\
e^{\omega_{2} \rho \pi} & e^{\omega_{1} \rho \pi} & e^{\omega_{2} \rho \pi}
\end{array}\right|=0 .
$$

Simple reductions give

$$
\Delta(\rho) \equiv 3-e^{3 \omega_{1} \rho \pi}-e^{3 \omega_{\rho} \rho \pi}-e^{3 \omega_{\rho} \rho \pi} \equiv-\delta_{1}(\rho \pi) \delta_{2}(\rho \pi) \delta_{3}(\rho \pi) .
$$

Accordingly, the characteristic numbers fall into three sets, all distributed along the positive axis of reals, and determined respectively by the equations

$$
\delta_{1}(\rho \pi)=0, \quad \delta_{2}(\rho \pi)=0, \quad \delta_{3}(\rho \pi)=0 .
$$

They are given respectively by

$$
\begin{gathered}
\rho_{1 k}=\frac{1}{3^{1 / 2}}(1+2 k)+\epsilon_{1 k}, \quad \rho_{2 k}=\frac{1}{3^{1 / 2}}\left(\frac{5}{3}+2 k\right)+\epsilon_{2 k}, \\
\rho_{3 k}=\frac{1}{3^{1 / 2}}\left(\frac{1}{3}+2 k\right)+\epsilon_{3 k},
\end{gathered}
$$

where $\epsilon_{i k}$ tends to zero as a limit when $k \rightarrow \infty, i$ being 1,2 , or 3 .

Use of the conditions $u(\pi)=u\left(-\omega_{2} \pi\right)=0$ gives

$$
\begin{aligned}
u(x)= & e^{\omega_{1} \rho x}\left(e^{2 \omega_{2} \rho x}-e^{-\omega_{2} \rho \pi}\right)+e^{\omega_{2} \rho x}\left(e^{2 \omega_{1} \rho x}-e^{-\omega_{1} \rho \pi}\right) \\
& +e^{\omega_{\rho} \rho x}\left(e^{2 \omega_{2} \rho \pi}-e^{-\omega_{2} \rho \pi}\right),
\end{aligned}
$$


except possibly for a factor independent of $x$. The characteristic numbers $\rho_{1 k}$ satisfy the following pair of equations; the second is obtained from the first by a rearrangement of terms:

Hence

$$
\begin{aligned}
& e^{\omega_{1} \rho \pi}+e^{\omega_{2} \rho \pi}+e^{\omega_{\rho} \rho x}=0, \\
& e^{\omega_{\rho} \rho \pi}+e^{\omega_{\rho} \rho \pi}+e^{\omega_{1} \rho \pi}=0 .
\end{aligned}
$$

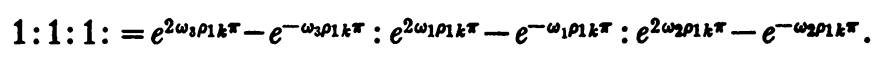

Thus we obtain from (42) for one set of characteristic functions $u_{1 k}(x)=\delta_{1}\left(\rho_{1 k} x\right)$, a suitable factor independent of $x$ having been divided out of the right hand side of (42). Similarly we obtain from (42) and the equations for the other sets of characteristic numbers $u_{2 k}(x)=\delta_{2}\left(\rho_{2 k} x\right)$ and $u_{3 k}(x)=\delta_{8}\left(\rho_{8 k} x\right)$.

We have thus three sets of characteristic functions; each set consists of the complete set of characteristic functions of an expansion problem of the type considered in Part I. Let $f(x)$ be a function analytic in $x$ at $x=0$. We may write $f(x)=\phi_{1}\left(x^{3}\right)+x \phi_{2}\left(x^{3}\right)+x^{2} \phi_{3}\left(x^{3}\right)$, each $\phi$ standing for a convergent power series in $x^{3}$. If each $\phi$ satisfies certain continuity conditions from 0 to $\pi$, Theorem 2 of Part I tells us that $x^{i-1} \phi_{i}\left(x^{3}\right)$ may be expanded in a series of type

$$
\sum_{k=1}^{\infty} a_{i k} u_{i k}(x)
$$$$
(i=1,2,3)
$$

We may, therefore, state

TheOREM 1. Subject to the continuity conditions on the interval $(0, \pi)$ of Theorem 2, Part I, any analytic function of $x$ may be expanded in a series of the type

$$
\sum_{1}^{\infty}\left[a_{1 k} u_{1 k}(x)+a_{2 k} u_{2 k}(x)+a_{3 k} u_{3 k}(x)\right],
$$

and the series will converge uniformly to the function in the interior of an equilateral triangle centered at $x=0$ and having one vertex on the segment from 0 to $\pi$.

The following theorem, which we will now prove, states essentially that a series of type (44) necessarily represents a function which is analytic in $x$.

TheOREM 2. If series (44) converges uniformly in an interval $(\alpha, \beta)$, $0 \leqq \alpha<\beta \leqq \pi$, each of the three series (43) converges uniformly in the interior of an equilateral triangle centered at $x=0$ and having one vertex in the interior of the interval $(\alpha, \beta)$. 
Let $x_{1 k}, x_{2 k}, x_{3 k}$ be three values of $x$ in $(\alpha, \beta), k$ being fixed, such that $u_{i k}\left(x_{i k}\right)=0, i=1,2,3$. It is clear that if $k$ is sufficiently large, three such values of $x$ will exist. Then, calling

we have

$$
u_{k}(x)=a_{1 k} u_{1 k}(x)+a_{2 k} u_{2 k}(x)+a_{3 k} u_{3 k}(x),
$$

Calling

we get

$$
\begin{aligned}
& u_{k}\left(x_{1 k}\right)=\quad a_{2 k} u_{2 k}\left(x_{1 k}\right)+a_{3 k} u_{3 k}\left(x_{1 k}\right), \\
& u_{k}\left(x_{2 k}\right)=a_{1 k} u_{1 k}\left(x_{2 k}\right) \quad+a_{3 k} u_{3 k}\left(x_{2 k}\right), \\
& u_{k}\left(x_{3 k}\right)=a_{1 k} u_{1 k}\left(x_{3 k}\right)+a_{2 k} u_{2 k}\left(x_{3 k}\right) .
\end{aligned}
$$

$$
\Delta \equiv\left|\begin{array}{ccc}
0 & u_{2 k}\left(x_{1 k}\right) & u_{3 k}\left(x_{1 k}\right) \\
u_{1 k}\left(x_{2 k}\right) & 0 & u_{3 k}\left(x_{2 k}\right) \\
u_{1 k}\left(x_{3 k}\right) & u_{2 k}\left(x_{3 k}\right) & 0
\end{array}\right|,
$$

$$
a_{1 k}=\frac{1}{\Delta}\left|\begin{array}{ccc}
u_{k}\left(x_{1 k}\right) & u_{2 k}\left(x_{1 k}\right) & u_{3 k}\left(x_{1 k}\right) \\
u_{k}\left(x_{2 k}\right) & 0 & u_{3 k}\left(x_{2 k}\right) \\
u_{k}\left(x_{3 k}\right) & u_{2 k}\left(x_{3 k}\right) & 0
\end{array}\right| \text {, }
$$

with similar formulas for $a_{2 k}$ and $a_{3 k}$. We shall use these expressions to determine the sizes of the $a$ 's.

We have

$$
u_{1 k}\left(x_{2 k}\right)=e^{-\rho_{1 k} x_{2 k}}+2 e^{\rho_{1 k} x_{2 k} / 2} \cos \left(\frac{3^{1 / 2}}{2} \rho_{1 k} x_{2 k}\right) .
$$

But from (41)

$$
\cos \left(\frac{3^{1 / 2}}{2} \rho_{1 k} x_{2 k}\right)=\cos \left[\frac{3^{1 / 2}}{2} \rho_{2 k} x_{2 k}-\frac{x_{2 k}}{3}+\frac{3^{1 / 2}}{2} x_{2 k}\left(\epsilon_{1 k}-\epsilon_{2 k}\right)\right] .
$$

Also, from $u_{2 k}\left(x_{2 k}\right)=0$, we get

whence

$$
\cos \left(-\frac{\pi}{3}+\frac{3^{1 / 2}}{2}-\rho_{2 k} x_{2 k}\right)=\frac{1}{2} e^{-3 \rho_{2 k} x_{2 k} / 2},
$$

$$
\sin \left(-\frac{\pi}{3}+\frac{3^{1 / 2}}{2} \rho_{2 k} x_{2 k}\right)=\left(1-\frac{1}{4} e^{-3 \rho_{2 k} x_{2 k} / 2}\right)^{1 / 2} .
$$

We may choose the positive sign before the radical by selecting $x_{2 k}$ properly. Hence

$$
\left(\cos \frac{3^{1 / 2}}{2} \rho_{1 k} x_{2 k}\right)=-\sin \left(\frac{\pi}{3}-\frac{x_{2 k}}{3}\right)+\epsilon,
$$

where $\epsilon$ is a symbol which we shall use for any quantity which can be made as small numerically as desired by taking $k$ large enough. Hence 


$$
u_{1 k}\left(x_{2 k}\right)=e^{\rho_{1 k} x_{2 k} / 2}\left[-2 \sin \left(\frac{\pi}{3}-\frac{x_{2 k}}{3}\right)+\epsilon\right]
$$

In a similar way we find

$$
\begin{aligned}
& u_{1 k}\left(x_{3 k}\right)=e^{\rho_{1 k} x_{3 k} / 2}\left[2 \sin \left(\frac{\pi}{3}-\frac{x_{3 k}}{3}\right)+\epsilon\right], \\
& u_{2 k}\left(x_{1 k}\right)=e^{\rho_{2 k} x_{1 k} / 2}\left[-2 \sin \left(\frac{\pi}{3}-\frac{x_{1 k}}{3}\right)+\epsilon\right], \\
& u_{2 k}\left(x_{3 k}\right)=e^{\rho_{2 k} x_{3 k} / 2}\left[-2 \sin \left(\frac{2 \pi}{3}-\frac{2 x_{3 k}}{3}\right)+\epsilon\right], \\
& u_{3 k}\left(x_{1 k}\right)=e^{\rho_{3 k} x_{1 k} / 2}\left[2 \sin \left(\frac{\pi}{3}-\frac{x_{1 k}}{3}\right)+\epsilon\right], \\
& u_{3 k}\left(x_{2 k}\right)=e^{\rho_{3 k} x_{2 k} / 2}\left[2 \sin \left(\frac{2 \pi}{3}-\frac{2 x_{2 k}}{3}\right)+\epsilon\right] .
\end{aligned}
$$

Hence

$$
\begin{aligned}
& \Delta=e^{\left(\rho_{1 k} x_{2 k}+\rho_{2 k} x_{3 k}+\rho_{3 k} x_{1 k}\right) / 2}\left.-2 \sin \left(\frac{\pi}{3}-\frac{x_{2 k}}{3}\right)+\epsilon\right] \\
& \cdot {\left[-2 \sin \left(\frac{2 \pi}{3}-\frac{2 x_{3 k}}{3}\right)+\epsilon\right]\left[2 \sin \left(\frac{\pi}{3}-\frac{x_{1 k}}{3}\right)+\epsilon\right] } \\
&+e^{\left(\rho_{1 k} x_{3 k}+\rho_{2 k} x_{1 k}+\rho_{3 k} x_{2 k}\right) / 2}\left[2 \sin \left(\frac{\pi}{3}-\frac{x_{3 k}}{3}\right)+\epsilon\right] \\
& \cdot\left[-2 \sin \left(\frac{\pi}{3}-\frac{x_{1 k}}{3}\right)+\epsilon\right]\left[2 \sin \left(\frac{2 \pi}{3}-\frac{2 x_{2 k}}{3}\right)+\epsilon\right]
\end{aligned}
$$

By means of obvious transformations this can be written

Now

$$
\begin{gathered}
\Delta=16 \sin \frac{\pi-x_{1 k}}{3} \sin \frac{\pi-x_{2 k}}{3} \sin \frac{\pi-x_{3 k}}{3} e^{\left(\rho_{1 k} x_{1 k}+\rho_{2 k} x_{2 k}+\rho_{2 k} x_{2 k}\right) / 2} \\
\cdot\left[\begin{array}{c}
e^{\left[\rho_{1 k}\left(x_{2 k}-x_{1 k}\right)+\rho_{2 k}\left(x_{3 k}-x_{2 k}\right)+\rho_{2 k}\left(x_{1 k}-x_{3 k}\right) / 2\right.}\left(\cos \frac{\pi-x_{3 k}}{3}+\epsilon\right) \\
-e^{\left[\rho_{1 k}\left(x_{2 k}-x_{1 k}\right)+\rho_{2 k}\left(x_{1 k}-x_{2 k}\right)+\rho_{2 k}\left(x_{2 k}-x_{3 k}\right) / 2\right.}\left(\cos \frac{\pi-x_{2 k}}{3}+\epsilon\right)
\end{array}\right] .
\end{gathered}
$$

$$
\begin{aligned}
\rho_{1 k}\left(x_{2 k}-x_{1 k}\right)+\rho_{2 k}\left(x_{3 k}-x_{2 k}\right)+\rho_{3 k}\left(x_{1 k}-x_{3 k}\right) & \\
& =\frac{2}{3 \cdot 3^{1 / 2}}\left(2 x_{3 k}-x_{1 k}-x_{2 k}\right)+\epsilon,
\end{aligned}
$$


and

$$
\begin{aligned}
\rho_{1 k}\left(x_{3 k}-x_{1 k}\right)+\rho_{2 k}\left(x_{1 k}-x_{2 k}\right)+\rho_{3 k}\left(x_{2 k}-x_{3 k}\right) & \\
& =\frac{2}{3 \cdot 3^{1 / 2}}\left(x_{1 k}+x_{3 k}-2 x_{2 k}\right)+\epsilon .
\end{aligned}
$$

We will make $2 x_{3 k}-x_{1 k}-x_{2 k}>x_{1 k}+x_{3 k}-2 x_{2 k}$ by taking $x_{2 k}+x_{3 k}>2 x_{1 k}$. To secure this we divide $(\alpha, \beta)$ into three pieces, each a closed interval and each separated from the others by some small fixed amount. We require $x_{1 k}$ to be chosen from the left hand one of these subintervals, and $x_{2 k}$ and $x_{3 k}$ from the other two. This renders the inequalities true. We will denote these three subintervals by I, II, and III in order from left to right. Evidently $\cos \left(\left(\pi-x_{3 k}\right) / 3\right)$ and $\cos \left(\left(\pi-x_{2 k}\right) / 3\right)$ are both positive. We require that $x_{2 k}$ and $x_{3 k}$ be chosen from intervals II and III respectively. This assures us that $\cos \left(\left(\pi-x_{3 k}\right) / 3\right)>\cos \left(\left(\pi-x_{2 k}\right) / 3\right)$. It is now clear, if $k$ is large enough, that

$$
|\Delta|>h e^{\left(\rho_{1 k} x_{1 k}+\rho_{2 k} x_{2 k}+\rho_{2 k} x_{3 k}\right) / 2} \text {, }
$$

where $h$ is independent of $k$.

Returning to (45) we have for the determinant there written out explicitly, which we shall call $N$,

$N=u_{k}\left(x_{2 k}\right) u_{2 k}\left(x_{3 k}\right) u_{3 k}\left(x_{1 k}\right)+u_{k}\left(x_{3 k}\right) u_{3 k}\left(x_{2 k}\right) u_{2 k}\left(x_{1 k}\right)-u_{k}\left(x_{1 k}\right) u_{2 k}\left(x_{3 k}\right) u_{3 k}\left(x_{2 k}\right)$.

On account of the assumed uniform convergence of series (44), $\left|u_{k}\left(x_{i k}\right)\right|$ is less than some number which is independent of $k$. Further,

$$
\begin{array}{ll}
\left|u_{2 k}\left(x_{2 k}\right)\right|<g e^{\rho_{2 k} x_{3 k} / 2}, & \left|u_{3 k}\left(x_{1 k}\right)\right|<g e^{\rho_{3 k} x_{1 k} / 2}, \\
\left|u_{3 k}\left(x_{2 k}\right)\right|<g e^{\rho_{3 k} x_{2 k} / 2}, & \left|u_{2 k}\left(x_{1 k}\right)\right|<g e^{\rho_{3 k} x_{1 k} / 2},
\end{array}
$$

the same $g$, which is independent of $k$, serving in all cases. Hence

$$
\begin{aligned}
& \left|u_{2 k}\left(x_{3 k}\right) u_{3 k}\left(x_{1 k}\right)\right|<g^{2} e^{\left(\rho_{2 k} x_{2 k}+\rho_{3 k} x_{1 k}\right) / 2}<\mu e^{\left(\rho_{2 k} x_{2 k}+\rho_{3 k} x_{3 k}\right) / 2}, \\
& \left|u_{3 k}\left(x_{2 k}\right) u_{2 k}\left(x_{1 k}\right)\right|<g^{2} e^{\left(\rho_{3 k} x_{2 k}+\rho_{2 k} x_{1 k}\right) / 2}<\mu e^{\left(\rho_{2 k} x_{2 k}+\rho_{3 k} x_{3 k}\right) / 2}, \\
& \left|u_{2 k}\left(x_{3 k}\right) u_{3 k}\left(x_{2 k}\right)\right|<g^{2} e^{\left(\rho_{2 k} x_{3 k}+\rho_{3 k} x_{2 k}\right) / 2}<\mu e^{\left(\rho_{2 k} x_{2 k}+\rho_{i k} x_{3 k}\right) / 2},
\end{aligned}
$$

$\mu$ being independent of $k$. These inequalities in conjunction with (46) yield $\left|a_{1 k}\right|<\nu_{1} e^{-\rho_{1 k} x_{1 k} / 2}$, where $\nu_{1}$ is independent of $k$.

In a similar way we find $\left|a_{2 k}\right|<\nu_{2} e^{-\rho_{2 k} x_{1 k} / 2}$ and $\left|a_{3 k}\right|<\nu_{3} e^{-\rho_{3 k} x_{1 k} / 2}$. Moreover it is clear that by taking $k$ sufficiently large we may choose $x_{1 k}$ so that $\beta-x_{1 k}$ is less than a preassigned positive constant. The theorem now follows by an argument like that used following the lemma of Part I. Evidently the triangle of this theorem may have one vertex as near to $\beta$ as is desired.

UNIERSITY OF IOWA, Iowa Ciry, Iowa 